\title{
Properties of magnetized neutral pions at zero and finite temperature in nonlocal chiral quark models
}

\author{
D. Gómez Dumm ${ }^{1,2}$ M. F. Izzo Villafañe ${ }^{2,3}$ and N. N. Scoccola ${ }^{2,3}$ \\ ${ }^{1}$ IFLP, CONICET-Departamento de Física, Facultad de Ciencias Exactas, \\ Universidad Nacional de La Plata, C.C. 67, (1900) La Plata, Argentina \\ ${ }^{2}$ CONICET, Rivadavia 1917, (1033) Buenos Aires, Argentina \\ ${ }^{3}$ Physics Department, Comisión Nacional de Energía Atómica, \\ Avenida del Libertador 8250, (1429) Buenos Aires, Argentina
}

(Received 24 April 2020; accepted 11 June 2020; published 29 June 2020)

\begin{abstract}
The behavior of $\pi^{0}$ meson properties in the presence of a uniform external magnetic field is studied in the context of a nonlocal extension of the Polyakov-Nambu-Jona-Lasinio model. The analysis includes the $\pi^{0}$ mass, the effective $\pi^{0}$-quark coupling and the pion-to-vacuum hadronic form factors, both at zero and finite temperature. Numerical results are compared with previous calculations carried out within the local NJL model, when available. The validity of chiral relations and the features of deconfinement and chiral symmetry restoration transitions are discussed.
\end{abstract}

DOI: $10.1103 /$ PhysRevD.101.116018

\section{INTRODUCTION}

The behavior of strongly interacting matter under the influence of intense magnetic fields has become an issue of increasing interest in the past few years (see [1-3] and references therein). This is mostly motivated by the realization that the presence of strong magnetic fields should be taken into account in the analysis of some relevant physical systems, e.g. in the description of high energy noncentral heavy ion collisions [4], or the study of magnetars [5]. From the theoretical point of view, addressing this subject requires to deal with quantum chromodynamics (QCD) in nonperturbative regimes. Therefore, present analyses are based either in the predictions of effective models (see e.g. Refs. [6-9]) or in the results obtained through lattice QCD (LQCD) calculations. In this work we focus on the effect of an intense external magnetic field on various $\pi^{0}$ meson properties at zero and finite temperature. This issue has been studied in the past years following various theoretical approaches for low-energy QCD, such as Nambu-Jona-Lasinio (NJL)-like models [10-17], chiral perturbation theory (ChPT) [18,19] and path integral Hamiltonians [20,21]. In addition, results for the light meson spectrum under background magnetic fields at zero temperature have been obtained from LQCD calculations [22,23].

Published by the American Physical Society under the terms of the Creative Commons Attribution 4.0 International license. Further distribution of this work must maintain attribution to the author(s) and the published article's title, journal citation, and DOI. Funded by SCOAP ${ }^{3}$.
In Ref. [24] we have studied the behavior of the $\pi^{0}$ meson mass and one of its axial decay form factors in the presence of a uniform static magnetic field at zero temperature, within a relativistic chiral quark model in which quarks interact through a nonlocal four-fermion coupling [25]. This so-called "nonlocal NJL (nINJL) model" can be viewed as a sort of extension of the NJL model that intends to provide a more realistic effective approach to QCD. Actually, nonlocality arises naturally in the context of successful descriptions of low-energy quark dynamics [26,27], and it has been shown [28] that nonlocal models can lead to a momentum dependence in quark propagators that is consistent with LQCD results. Moreover, in this framework it is possible to obtain an adequate description of the properties of light mesons in the absence of an external electromagnetic field at both zero and finite temperature [28-39]. Interestingly, as shown in Refs. [40,41], nINJL models naturally allow to reproduce the so-called inverse magnetic catalysis (IMC) effect, previously observed from LQCD results. According to these calculations, the chiral restoration critical temperature turns out to be a decreasing function of the magnetic field $B$. In fact, the observation of IMC in LQCD calculations $[42,43]$ represents a challenge from the point of view of theoretical models, since most naive effective approaches to low energy QCD (NJL model, ChPT, MIT bag model, quark-meson models) predict that the chiral transition temperature should grow when the magnetic field is increased [1-3]. In addition, nlNJL models lead to a $B$ dependence of the $\pi^{0}$ mass that is found to be in good agreement with LQCD results [24]. 
The aim of the present article is to extend the work in Ref. [24], considering some additional properties of the magnetized $\pi^{0}$ mesons. As shown in Ref. [44], in the presence of a constant magnetic field $\vec{B}$ the pion-to-vacuum vector and axial vector amplitudes can be in general parametrized in terms of three "decay" form factors. One of them, $f_{\pi^{0}}^{(A \|)}$, corresponds to the pion decay constant usually denoted by $f_{\pi}$. The behavior of this form factor under the magnetic field has already been analyzed in Ref. [24], together with those of the masses $m_{\pi^{0}}$ and $m_{\sigma}$, and the quark-meson coupling $g_{\pi^{0} q q}$. The other two decay form factors are a second axial decay constant, $f_{\pi^{0}}^{(A \perp)}$, associated to momentum components that are perpendicular to the magnetic field, and a vector decay constant $f_{\pi^{0}}^{(V)}$. As shown in Ref. [10], another relevant feature induced by the presence of the external magnetic field is the fact that the $\pi^{0}$ dispersion relation turns out to be anisotropic, implying that the movement along the direction perpendicular to the magnetic field is characterized by a diffraction index $u_{\pi^{0}}$ which is in general different from one. In this way, to complement the analysis carried out in Ref. [24], in this work we study the magnetic field dependence of $f_{\pi^{0}}^{(A \perp)}, f_{\pi^{0}}^{(V)}$ and $u_{\pi^{0}}$. In addition, we extend the analysis to a system at finite temperature $T$, considering the thermal behavior of these quantities and also of the masses $m_{\pi^{0}}$ and $m_{\sigma}$, the coupling $g_{\pi^{0} q q}$ and the decay constant $f_{\pi^{0}}^{(A \|)}$, which have been studied in Ref. [24] only for $T=0$.

This article is organized as follows. In Sec. II we show how to obtain the analytical equations required to determine the relevant $\pi^{0}$ properties at zero temperature in the presence of the magnetic field. Our calculations are based on the formalism developed in Refs. [24,40,41], which make use of Ritus eigenfunctions [45]. In Sec. III we show how to extend the analysis in Sec. II to a system at finite temperature, taking also into account the coupling of fermions to a background color field (the so-called "Polyakov loop nlNJL model"). In Sec. IV we quote and discuss our numerical results, while in Sec. V we present our conclusions. Finally, in Appendixes A and B we outline the derivation of some of the expressions quoted in the main text.

\section{THEORETICAL FORMALISM}

The Euclidean action for the nonlocal NJL-like twoflavor quark model we are considering reads

$S_{E}=\int d^{4} x\left\{\bar{\psi}(x)\left(-i \not \partial+m_{c}\right) \psi(x)-\frac{G}{2} j_{a}(x) j_{a}(x)\right\}$.

Here $m_{c}$ is the current quark mass, equal for $u$ and $d$ quarks, while the currents $j_{a}(x)$ are given by

$$
j_{a}(x)=\int d^{4} z \mathcal{G}(z) \bar{\psi}\left(x+\frac{z}{2}\right) \Gamma_{a} \psi\left(x-\frac{z}{2}\right),
$$

where $\Gamma_{a}=\left(\mathbb{1}, i \gamma_{5} \vec{\tau}\right)$. The function $\mathcal{G}(z)$ is a nonlocal form factor that characterizes the effective interaction. The action can be "gauged" to incorporate couplings to electromagnetic, vector and axial vector gauge fields $\mathcal{A}_{\mu}, W_{\mu}^{V, a}(x)$ and $W_{\mu}^{A, a}(x)$, respectively. This is done by replacing

$$
\partial_{\mu} \rightarrow D_{\mu} \equiv \partial_{\mu}-i \hat{Q} \mathcal{A}_{\mu}(x)-\frac{i}{2} \Gamma^{C} \tau^{a} W_{\mu}^{C, a}(x),
$$

where $\hat{Q}=\operatorname{diag}\left(q_{u}, q_{d}\right)$, with $q_{u}=2 e / 3, q_{d}=-e / 3$, $C=V, A, a=1,2,3, \Gamma^{V}=1$ and $\Gamma^{A}=\gamma_{5}$. For this nonlocal model, gauge symmetry also requires the replacements $[28,34,39]$

$$
\begin{aligned}
\psi(x-z / 2) & \rightarrow \mathcal{W}(x, x-z / 2) \psi(x-z / 2), \\
\psi(x+z / 2)^{\dagger} & \rightarrow \psi(x+z / 2)^{\dagger} \mathcal{W}(x+z / 2, x),
\end{aligned}
$$

with

$$
\begin{aligned}
\mathcal{W}(x, y)= & \exp \left[-i\left(\hat{Q} \int_{x}^{y} d \ell_{\mu} A_{\mu}(\ell)\right.\right. \\
& \left.\left.+\frac{\tau^{a}}{2} \Gamma^{C} \int_{x}^{y} d \ell_{\mu} W_{\mu}^{C, a}(\ell)\right)\right],
\end{aligned}
$$

where $\ell$ runs over an arbitrary path connecting $x$ with $y$. As it is usually done, we take it to be a straight line path.

As stated, we assume the presence of an external uniform magnetic field $\vec{B}$. Therefore, using the Landau gauge, and choosing the $x_{3}$ axis in the direction of $\vec{B}$, we take $\mathcal{A}_{\mu}$ to be a static field given by $\mathcal{A}_{\mu}(x)=B x_{1} \delta_{\mu 2}$.

Since we are interested in studying light meson properties, we carry out a bosonization of the fermionic theory, introducing scalar and pseudoscalar fields $\sigma(x)$ and $\vec{\pi}(x)$ and integrating out the fermion fields. The bosonized action can be written as $[24,28,39]$

$S_{\text {bos }}=-\log \operatorname{det} \mathcal{D}+\frac{1}{2 G} \int d^{4} x[\sigma(x) \sigma(x)+\vec{\pi}(x) \cdot \vec{\pi}(x)]$,

where

$$
\begin{aligned}
\mathcal{D}\left(x, x^{\prime}\right)= & \delta^{(4)}\left(x-x^{\prime}\right)\left(-i \not D+m_{c}\right) \\
& +\mathcal{G}\left(x-x^{\prime}\right) \gamma_{0} \mathcal{W}(x, \bar{x}) \gamma_{0}[\sigma(\bar{x}) \\
& \left.+i \gamma_{5} \vec{\tau} \cdot \vec{\pi}(\bar{x})\right] \mathcal{W}\left(\bar{x}, x^{\prime}\right)
\end{aligned}
$$

with $\bar{x}=\left(x+x^{\prime}\right) / 2$. We expand now the meson fields around their mean field values. Since the external magnetic field is uniform, one can assume that the field $\sigma(x)$ has a 
nontrivial translational invariant mean field value $\bar{\sigma}$, while the vacuum expectation values of pseudoscalar fields are zero. We separate the mean field piece of the first term of the action in Eq. (6), writing

$$
-\log \operatorname{det} \mathcal{D}=-\operatorname{Tr} \log \mathcal{D}_{0}-\operatorname{Tr} \log \left(1+\mathcal{D}_{0}^{-1} \delta \mathcal{D}\right),
$$

where the traces run over color, flavor, Dirac and coordinate spaces. The form of the mean field operator $\mathcal{D}_{0}$ in the presence of the external magnetic field has been studied in detail in previous works, see e.g. Ref. [41]. It can be written as

$$
\mathcal{D}_{0}=\operatorname{diag}\left(\mathcal{D}_{u}^{\mathrm{MFA}}\left(x, x^{\prime}\right), \mathcal{D}_{d}^{\mathrm{MFA}}\left(x, x^{\prime}\right)\right),
$$

where

$$
\begin{aligned}
\mathcal{D}_{f}^{\mathrm{MFA}}\left(x, x^{\prime}\right)= & \delta^{(4)}\left(x-x^{\prime}\right)\left(-i \not \partial-q_{f} B x_{1} \gamma_{2}+m_{c}\right) \\
& +\bar{\sigma} \mathcal{G}\left(x-x^{\prime}\right) \exp \left[i \Phi_{f}\left(x, x^{\prime}\right)\right] .
\end{aligned}
$$

Here $\Phi_{f}\left(x, x^{\prime}\right)=q_{f} B\left(x_{2}-x_{2}^{\prime}\right)\left(x_{1}+x_{1}^{\prime}\right) / 2$ is the so-called Schwinger phase, and a direct product to an identity matrix in color space is understood. The mean field quark propagators $S_{f}^{\mathrm{MFA}}\left(x, x^{\prime}\right)=\left[\mathcal{D}_{f}^{\mathrm{MFA}}\left(x, x^{\prime}\right)\right]^{-1}$ can be obtained following the Ritus eigenfunction method [45]. As shown in Ref. [41] (see also the analysis carried out within the Schwinger-Dyson formalism in Refs. [46,47]), it is possible to write the propagators in terms of the Schwinger phase and a translational invariant function, namely

$S_{f}^{\mathrm{MFA}}\left(x, x^{\prime}\right)=\exp \left[i \Phi_{f}\left(x, x^{\prime}\right)\right] \int \frac{d^{4} p}{(2 \pi)^{4}} e^{i p \cdot\left(x-x^{\prime}\right)} \tilde{S}_{f}\left(p_{\perp}, p_{\|}\right)$,

where $p_{\perp}=\left(p_{1}, p_{2}\right)$ and $p_{\|}=\left(p_{3}, p_{4}\right)$. The expression of $\tilde{S}_{f}\left(p_{\perp}, p_{\|}\right)$in the nlNJL model under consideration is found to be [41]

$$
\begin{aligned}
\tilde{S}_{f}\left(p_{\perp}, p_{\|}\right)= & 2 \exp \left(-p_{\perp}^{2} /\left|q_{f} B\right|\right) \sum_{k=0}^{\infty} \sum_{\lambda= \pm}\left[(-1)^{k_{\lambda}}\right. \\
& \times\left(\hat{A}_{k, p_{\|}}^{\lambda, f}-\hat{B}_{k, p_{\|}}^{\lambda, f} p_{\|} \cdot \gamma_{\|}\right) L_{k_{\lambda}}\left(2 p_{\perp}^{2} /\left|q_{f} B\right|\right) \\
& +2(-1)^{k}\left(\hat{C}_{k, p_{\|}}^{\lambda, f}-\hat{D}_{k, p_{\|}}^{\lambda, f} p_{\|} \cdot \gamma_{\|}\right) \\
& \left.\times p_{\perp} \cdot \gamma_{\perp} L_{k-1}^{1}\left(2 p_{\perp}^{2} /\left|q_{f} B\right|\right)\right] \Delta^{\lambda},
\end{aligned}
$$

where the following definitions have been used. The perpendicular and parallel gamma matrices are collected in vectors $\gamma_{\perp}=\left(\gamma_{1}, \gamma_{2}\right)$ and $\gamma_{\|}=\left(\gamma_{3}, \gamma_{4}\right)$, while the matrices $\Delta^{\lambda}$ are defined as $\Delta^{+}=\operatorname{diag}(1,0,1,0)$ and $\Delta^{-}=$ $\operatorname{diag}(0,1,0,1)$. The integers $k_{\lambda}$ are given by $k_{ \pm}=$ $k-1 / 2 \pm s_{f} / 2$, where $s_{f}=\operatorname{sign}\left(q_{f} B\right)$. The functions $\hat{X}_{k, p_{\|}}^{ \pm, f}$, with $X=A, B, C, D$, are defined as

$$
\begin{gathered}
\hat{A}_{k, p_{\|}}^{ \pm, f}=M_{k, p_{\|}}^{\mp, f} \hat{C}_{k, p_{\|}}^{ \pm, f}+p_{\|}^{2} \hat{D}_{k, p_{\|}}^{ \pm, f}, \\
\hat{B}_{k, p_{\|}}^{ \pm, f}=\hat{C}_{k, p_{\|}}^{ \pm, f}-M_{k, p_{\|}}^{\mp, f} \hat{D}_{k, p_{\|}}^{ \pm, f}, \\
\hat{C}_{k, p_{\|}}^{ \pm, f}=\frac{2 k\left|q_{f} B\right|+p_{\|}^{2}+M_{k, p_{\|}}^{-, f} M_{k, p_{\|}}^{+, f}}{\Delta_{k, p_{\|}}^{f}}, \\
\hat{D}_{k, p_{\|}}^{ \pm, f}=\frac{M_{k, p_{\|}}^{ \pm, f}-M_{k, p_{\|}}^{\mp, f}}{\Delta_{k, p_{\|}}^{f}},
\end{gathered}
$$

where

$\Delta_{k, p_{\|}}^{f}=\left(2 k\left|q_{f} B\right|+p_{\|}^{2}+M_{k, p_{\|}}^{+, f} M_{k, p_{\|}}^{-, f}\right)^{2}+p_{\|}^{2}\left(M_{k, p_{\|}}^{+, f}-M_{k, p_{\|}}^{-, f}\right)^{2}$.

The functions $M_{k, p_{\|}}^{\lambda, f}$ play the role of effective (momentumdependent) dynamical quark masses in the presence of the magnetic field. They are given by

$$
\begin{aligned}
M_{k, p_{\|}}^{\lambda, f}= & \frac{4 \pi}{\left|q_{f} B\right|}(-1)^{k_{\lambda}} \int \frac{d^{2} p_{\perp}}{(2 \pi)^{2}} M\left(p_{\perp}^{2}+p_{\|}^{2}\right) \\
& \times \exp \left(-p_{\perp}^{2} /\left|q_{f} B\right|\right) L_{k_{\lambda}}\left(2 p_{\perp}^{2} /\left|q_{f} B\right|\right),
\end{aligned}
$$

where

$$
M\left(p^{2}\right)=m_{c}+\bar{\sigma} g\left(p^{2}\right)
$$

$g\left(p^{2}\right)$ being the Fourier transform of the nonlocal form factor $\mathcal{G}(x)$. In Eqs. (12) and (18), $L_{k}(x)$ and $L_{k}^{1}(x)$ stand for generalized Laguerre polynomials, with the convention $L_{-1}(x)=L_{-1}^{1}(x)=0$. The relation in Eq. (18) can be understood as a Laguerre-Fourier transform of the function $M\left(p^{2}\right)$. It is also convenient to introduce the LaguerreFourier transform of the form factor $g\left(p^{2}\right)$,

$$
\begin{aligned}
g_{k, p_{\|}}^{\lambda, f}= & \frac{4 \pi}{\left|q_{f} B\right|}(-1)^{k_{\lambda}} \int \frac{d^{2} p_{\perp}}{(2 \pi)^{2}} g\left(p_{\perp}^{2}+p_{\|}^{2}\right) \\
& \times \exp \left(-p_{\perp}^{2} /\left|q_{f} B\right|\right) L_{k_{\lambda}}\left(2 p_{\perp}^{2} /\left|q_{f} B\right|\right),
\end{aligned}
$$

thus one has

$$
M_{k, p_{\|}}^{\lambda, f}=\left[1-\delta_{\left(k_{\lambda}+1\right) 0}\right] m_{c}+\bar{\sigma} g_{k, p}^{\lambda, f} .
$$

The transform in Eq. (20) can be inverted to get 
$g\left(p_{\perp}^{2}+p_{\|}^{2}\right)=2 e^{-p_{\perp}^{2} /\left|q_{f} B\right|} \sum_{k=0}^{\infty}(-1)^{k_{\lambda}} g_{k, p_{\|}}^{\lambda, f} L_{k_{\lambda}}\left(2 p_{\perp}^{2} /\left|q_{f} B\right|\right)$.

To study the mass and decay form factors of the neutral pion, we expand the operator $\delta \mathcal{D}\left(x, x^{\prime}\right)$ in powers of the meson fluctuations and the external vector and axial vector fields, keeping up to linear terms in $\delta \pi_{3}, W_{\mu}^{V, 3}$ and $W_{\mu}^{A, 3}$. We obtain

$$
\begin{aligned}
\delta \mathcal{D}\left(x, x^{\prime}\right)= & \delta \mathcal{D}_{\pi}\left(x, x^{\prime}\right)+\delta \mathcal{D}_{W}^{(a)}\left(x, x^{\prime}\right)+\delta \mathcal{D}_{W}^{(b)}\left(x, x^{\prime}\right) \\
& +\delta \mathcal{D}_{W, \pi}\left(x, x^{\prime}\right),
\end{aligned}
$$

where

$$
\begin{aligned}
\delta \mathcal{D}_{\pi}\left(x, x^{\prime}\right)= & i \gamma_{5} \tau^{0} \exp \left[i \Phi\left(x, x^{\prime}\right)\right] g\left(x-x^{\prime}\right) \delta \pi_{3}(\bar{x}) \\
\delta \mathcal{D}_{W}^{(a)}\left(x, x^{\prime}\right)= & -\delta^{(4)}\left(x-x^{\prime}\right) \frac{\tau^{3}}{2} \sum_{C=V, A} \bar{\Gamma}^{C} \gamma_{\mu} W_{\mu}^{C, 3}(\bar{x}) \\
\delta \mathcal{D}_{W}^{(b)}\left(x, x^{\prime}\right)= & i \sigma \frac{\tau^{3}}{2} \exp \left[i \Phi\left(x, x^{\prime}\right)\right] g\left(x-x^{\prime}\right) \\
& \times \sum_{C=V, A} \bar{\Gamma}^{C}\left[U^{C, 3}(x, \bar{x})-U^{C, 3}\left(\bar{x}, x^{\prime}\right)\right] \\
\delta \mathcal{D}_{W, \pi}\left(x, x^{\prime}\right)= & -\frac{1}{2} \exp \left[i \Phi\left(x, x^{\prime}\right)\right] g\left(x-x^{\prime}\right) \\
& \times \sum_{C=V, A} \gamma_{5} \Gamma^{C}\left[U^{C, 3}(x, \bar{x})-U^{C, 3}\left(\bar{x}, x^{\prime}\right)\right] \delta \pi_{3}(\bar{x}) .
\end{aligned}
$$

Here we have used the definitions $\bar{x}=\left(x+x^{\prime}\right) / 2, \bar{\Gamma}^{C}=$ $\gamma_{0} \Gamma^{C} \gamma_{0}$ and

$$
U^{C, 3}(x, y)=\int_{x}^{y} d \ell_{\mu} W_{\mu}^{C, 3}(\ell) .
$$

Given a definite model parametrization, the value of $\bar{\sigma}$ can be found by minimization of the effective action at the mean field level. The corresponding "gap equation" reads $[40,41]$

$$
\frac{\bar{\sigma}}{G}=\frac{N_{C}}{\pi} \sum_{f=u, d} B_{f} \sum_{k=0}^{\infty} \int_{q_{\|}} \sum_{\lambda= \pm} g_{k, q_{\|}}^{\lambda, f} \hat{A}_{k, p}^{\lambda, f} .
$$

\section{A. Pion field redefinition and quark-meson coupling constants}

The calculation of the $\pi^{0}$ mass in this model has been previously carried out in Ref. [24]. As shown in that paper, the piece of the bosonized action that is quadratic in the neutral pion fields can be written as

$$
\begin{aligned}
\left.S_{\text {bos }}\right|_{\left(\delta \pi_{3}\right)^{2}} & =\left.\frac{1}{2} \operatorname{Tr}\left(\mathcal{D}_{0}^{-1} \delta \mathcal{D}_{\pi}\right)^{2}\right|_{\left(\delta \pi_{3}\right)^{2}}+\frac{1}{2 G} \int_{t_{\perp} t_{\|}} \delta \pi_{3}(t) \delta \pi_{3}(-t) \\
& =\frac{1}{2} \int_{t_{\perp} t_{\|}}\left[F\left(t_{\perp}^{2}, t_{\|}^{2}\right)+\frac{1}{G}\right] \delta \pi_{3}(t) \delta \pi_{3}(-t)
\end{aligned}
$$

where for integration in two-component momentum spaces we use the notation

$$
\int_{p q r \ldots} \equiv \int \frac{d^{2} p}{(2 \pi)^{2}} \frac{d^{2} q}{(2 \pi)^{2}} \frac{d^{2} r}{(2 \pi)^{2}} \cdots
$$

Choosing the frame in which the $\pi^{0}$ meson is at rest, its mass can be obtained as the solution of the equation

$$
\frac{1}{G}+F\left(0,-m_{\pi^{0}}^{2}\right)=0 .
$$

To normalize the pion field we can expand the action in Eq. (30) around the pion pole $\left(t_{\perp}=0, t_{\|}^{2}=-m_{\pi^{0}}^{2}\right)$ up to first order in momentum squared. We define

$$
\begin{aligned}
& Z_{\|}^{-1}=\left.\frac{d F\left(t_{\perp}^{2}, t_{\|}^{2}\right)}{d t_{\|}^{2}}\right|_{t_{\perp}^{2}=0, t_{\|}^{2}=-m_{\pi^{2}}^{2}}, \\
& Z_{\perp}^{-1}=\left.\frac{d F\left(t_{\perp}^{2}, t_{\|}^{2}\right)}{d t_{\perp}^{2}}\right|_{t_{\perp}^{2}=0, t_{\|}^{2}=-m_{\pi^{0}}^{2}},
\end{aligned}
$$

and renormalize the pion field according to $\pi_{3}(q)=$ $g_{\pi^{0} q q} \tilde{\pi}_{3}(q)$, where $g_{\pi^{0} q q}=Z_{\|}^{1 / 2}$ is the meson-quark effective coupling constant. Thus, one has

$S_{\pi^{0}}^{\text {quad }}=\frac{1}{2} \int_{q_{\perp} q_{\|}} \delta \tilde{\pi}_{3}(-q)\left(u_{\pi^{0}}^{2} q_{\perp}^{2}+q_{\|}^{2}+m_{\pi^{0}}^{2}\right) \delta \tilde{\pi}_{3}(q)$,

where

$$
u_{\pi^{0}}^{2}=\frac{Z_{\|}}{Z_{\perp}} .
$$

From the above expressions of the quark propagators and $\delta \mathcal{D}_{\pi}$, after some straightforward calculation we find

$$
\begin{aligned}
F\left(t_{\perp}^{2}, t_{\|}^{2}\right)= & -16 \pi^{2} N_{C} \sum_{f=u, d} \frac{1}{\left(q_{f} B\right)^{2}} \int_{q_{\perp} p_{\perp} p_{\perp}^{\prime} q_{\|}} g\left(q_{\perp}^{2}+q_{\|}^{2}\right) g\left[\left(p_{\perp}^{\prime}+p_{\perp}-q_{\perp}\right)^{2}+q_{\|}^{2}\right] \\
& \times \exp \left[i 2 \phi\left(q_{\perp}, p_{\perp}, p_{\perp}^{\prime}, t_{\perp}\right) /\left(q_{f} B\right)\right] \operatorname{tr}_{D}\left[\tilde{S}_{f}\left(p_{\perp}, q_{\|}^{+}\right) \gamma_{5} \tilde{S}_{f}\left(p_{\perp}^{\prime}, q_{\|}^{-}\right) \gamma_{5}\right],
\end{aligned}
$$

where the trace is taken over Dirac space. We have defined $q_{\|}^{ \pm}=q_{\|} \pm t_{\|} / 2$, while the function $\phi$ in the exponential is given by 
$\phi\left(q_{\perp}, p_{\perp}, p_{\perp}^{\prime}, t_{\perp}\right)=p_{2} p_{1}^{\prime}+q_{1}\left(p_{2}^{\prime}-p_{2}\right)-p_{1} p_{2}^{\prime}-q_{2}\left(p_{1}^{\prime}-p_{1}\right)+t_{2}\left(q_{1}-\left(p_{1}+p_{1}^{\prime}\right) / 2\right)-t_{1}\left(q_{2}-\left(p_{2}+p_{2}^{\prime}\right) / 2\right)$.

As stated in Ref. [24], the trace in Eq. (36) is given by

$$
\begin{aligned}
& \operatorname{tr}_{D}\left[\tilde{S}_{f}\left(p_{\perp}, q_{\|}^{+}\right) \gamma_{5} \tilde{S}_{f}\left(p_{\perp}^{\prime}, q_{\|}^{-}\right) \gamma_{5}\right]=8 e^{-\left(p_{\perp}^{2}+p_{\perp}^{\prime 2}\right) / B_{f}} \sum_{k, k^{\prime}=0}^{\infty}(-1)^{k+k^{\prime}}\left[\sum_{\lambda= \pm} F_{k k^{\prime}, q_{\|}^{+} q_{\|}^{-}}^{\lambda, f(A B)} L_{k_{\lambda}}\left(2 p_{\perp}^{2} / B_{f}\right) L_{k_{\lambda}^{\prime}}\left(2 p_{\perp}^{\prime 2} / B_{f}\right)\right. \\
& \left.+8 F_{k k^{\prime}, q_{\|}^{+} q_{\|}^{-}}^{+, f(C D)}\left(p_{\perp} \cdot p_{\perp}^{\prime}\right) L_{k-1}^{1}\left(2 p_{\perp}^{2} / B_{f}\right) L_{k^{\prime}-1}^{1}\left(2 p_{\perp}^{\prime 2} / B_{f}\right)\right]
\end{aligned}
$$

with

$$
F_{k k^{\prime}, q_{\|}^{+} q_{\|}^{-}}^{\lambda, f(X Y)}=\hat{X}_{k, q_{\|}^{+}}^{\lambda, f} \hat{X}_{k^{\prime}, q_{\|}^{-}}^{\lambda, f}+\left(q_{\|}^{+} \cdot q_{\|}^{-}\right) \hat{Y}_{k, q_{\|}^{+}}^{\lambda, f} \hat{Y}_{k^{\prime}, q_{\|}^{-}}^{\lambda, f}
$$

For simplicity we use the notation $B_{f}=\left|q_{f} B\right|$.

To work out the integrals in Eq. (36) it is convenient to use the Laguerre-Fourier transforms introduced above. In this way, the integrals over perpendicular momenta can be performed analytically. As found in Ref. [24], the expression for $F\left(0,-m_{\pi^{0}}^{2}\right)$ is

$$
F\left(0,-m_{\pi^{0}}^{2}\right)=-\frac{N_{C}}{\pi} \sum_{f=u, d} B_{f} \sum_{k=0}^{\infty} \int_{q_{\|}} \sum_{\lambda= \pm} g_{k, q_{\|}}^{\lambda, f}\left[g_{k, q_{\|}}^{\lambda, f} F_{k k, q_{\|}^{+} q_{\|}^{-}}^{\lambda, f(A B)}+\left.2 k B_{f} g_{k, q_{\|}}^{-\lambda, f} F_{k k, q_{\|}^{+} q_{\|}^{-}}^{\lambda, f(C D)}\right|_{t_{\|}^{2}=-m_{\pi^{0}}^{2}} .\right.
$$

The normalization constant $Z_{\|}^{-1}$ can be obtained by derivation on the rhs with respect to $t_{\|}^{2}$.

To obtain an expression for $Z_{\perp}^{-1}$ one has to expand $F\left(t_{\perp}^{2}, t_{\|}^{2}\right)$ up to first order in $t_{\perp}^{2}$. The calculation of the corresponding integrals over perpendicular momenta is sketched in Appendix A. One finally gets

$$
\begin{aligned}
Z_{\perp}^{-1}= & \frac{N_{C}}{4 \pi} \sum_{f=u, d} \sum_{k=0}^{\infty} \int_{q_{\|}} \sum_{\lambda= \pm}\left\{\left(g_{k, q_{\|}}^{\lambda, f} F_{k k, q_{\|}^{+} q_{\|}^{-}}^{\lambda, f(A B)}+2 k B_{f} g_{k, q_{\|}}^{-\lambda, f} F_{k k, q_{\|}^{+} q_{\|}^{-}}^{\lambda, f(C D)}\right)\right. \\
& \times\left[k_{\lambda}\left(g_{k-1, q_{\|}}^{\lambda, f}+g_{k, q_{\|}}^{\lambda, f}\right)+\left(k_{\lambda}+1\right)\left(g_{k, q_{\|}}^{\lambda, f}+g_{k+1, q_{\|}}^{\lambda, f}\right)\right]-\left(g_{k-1, q_{\|}}^{\lambda, f}+g_{k, q_{\|}}^{\lambda, f}\right) \\
& \times\left[k_{\lambda}\left(g_{k-1, q_{\|}}^{\lambda, f}+g_{k, q_{\|}}^{\lambda, f}\right) F_{k k-1, q_{\|}^{+} q_{\|}^{-}}^{\lambda, f(A B)}+2 B_{f} k(k-1)\left(g_{k-1, q_{\|}}^{-\lambda, f}+g_{k, q_{\|}}^{-\lambda, f}\right) F_{k k-1, q_{\|}^{+} q_{\|}^{-}}^{\lambda, f(C D)}\right] .
\end{aligned}
$$

\section{B. $\pi^{0}$ decay form factors}

The $\pi^{0}$-to-vacuum amplitudes for vector and axial vector quark currents are given by

$$
H_{\mu}^{V, 0}(x, \vec{p})=\left\langle 0\left|\bar{\psi}(x) \gamma_{\mu} \frac{\tau^{3}}{2} \psi(x)\right| \pi_{0}(\vec{p})\right\rangle, \quad H_{\mu}^{A, 0}(x, \vec{p})=\left\langle 0\left|\bar{\psi}(x) \gamma_{\mu} \gamma_{5} \frac{\tau^{3}}{2} \psi(x)\right| \pi_{0}(\vec{p})\right\rangle .
$$

As discussed in Ref. [44], in the presence of an external magnetic field these currents can be written in terms of three form factors. Following the notation in Ref. [48], in Euclidean space we have

$$
\begin{aligned}
& H_{4}^{V, 0}(x, \vec{p}) \pm H_{3}^{V, 0}(x, \vec{p})=\mp f_{\pi^{0}}^{(V)}\left(p_{4} \mp p_{3}\right) e^{i p \cdot x} \\
& H_{1}^{V, 0}(x, \vec{p}) \pm i H_{2}^{V, 0}(x, \vec{p})=0 \\
& H_{4}^{A, 0}(x, \vec{p}) \pm H_{3}^{A, 0}(x, \vec{p})=-i f_{\pi^{0}}^{(A \|)}\left(p_{4} \pm p_{3}\right) e^{i p \cdot x} \\
& H_{1}^{A, 0}(x, \vec{p}) \pm i H_{2}^{A, 0}(x, \vec{p})=-i f_{\pi^{0}}^{(A \perp)}\left(p_{1} \pm i p_{2}\right) e^{i p \cdot x}
\end{aligned}
$$

If we write the corresponding piece of the bosonic action as

$$
\left.S_{\mathrm{bos}}\right|_{W^{3} \delta \pi_{3}}=\sum_{C=V, A} \int_{t_{\|} t_{\perp}} F_{\mu}^{C}(t) W_{\mu}^{C, 3}(t) \delta \pi_{3}(-t)
$$

it is easily seen that 


$$
\begin{gathered}
f_{\pi^{0}}^{(V)}=\frac{Z_{\|}^{1 / 2}}{t_{\|}^{2}}\left[t_{3} F_{4}^{V}(t)-t_{4} F_{3}^{V}(t)\right], \\
f_{\pi^{0}}^{(A \|)}=i \frac{Z_{\|}^{1 / 2}}{t_{\|}^{2}} t_{\|} \cdot F_{\|}^{A}(t), \\
f_{\pi^{0}}^{(A \perp)}=i \frac{Z_{\|}^{1 / 2}}{t_{\perp}^{2}} t_{\perp} \cdot F_{\perp}^{A}(t) .
\end{gathered}
$$

The functions $F_{\mu}^{C}(t)$ can be separated into three pieces $F_{\mu}^{C,(i)}(t)$ with $i=\mathrm{I}$, II, III, coming from the various contributions to the effective action, namely

$$
\begin{gathered}
\left.S_{\text {bos }}^{\mathrm{I}}\right|_{W \delta \pi}=-\operatorname{Tr}\left[\mathcal{D}_{0}^{-1} \delta \mathcal{D}_{W, \pi}\right], \\
\left.S_{\text {bos }}^{\text {II }}\right|_{W \delta \pi}=\operatorname{Tr}\left[\mathcal{D}_{0}^{-1} \delta \mathcal{D}_{W}^{(a)} \mathcal{D}_{0}^{-1} \delta \mathcal{D}_{\pi}\right], \\
\left.S_{\text {bos }}^{\text {III }}\right|_{W \delta \pi}=\operatorname{Tr}\left[\mathcal{D}_{0}^{-1} \delta \mathcal{D}_{W}^{(b)} \mathcal{D}_{0}^{-1} \delta \mathcal{D}_{\pi}\right] .
\end{gathered}
$$

$$
\begin{aligned}
& F_{\mu}^{C,(\mathrm{I})}(t)=\frac{N_{C}}{2} \sum_{f=u, d} \int_{q_{\|} r_{\|} q_{\perp} r_{\perp}}\left[g\left((q-r / 2)^{2}\right)-g\left((q-r / 2+t / 2)^{2}\right)\right] \operatorname{tr}_{D}\left[\tilde{S}_{f}\left(q_{\perp}, q_{\|}\right) \gamma_{5} \Gamma^{C}\right] h_{\mu}(r, t-r), \\
& F_{\mu}^{C,(\mathrm{II})}(t)=-i 8 \pi^{2} N_{C} \sum_{f=u, d} \frac{1}{B_{f}^{2}} \int_{q_{\|} q_{\perp} p_{\perp} p_{\perp}^{\prime}} g\left(q^{2}\right) \exp \left[i 2 \varphi\left(q_{\perp}, p_{\perp}, p_{\perp}^{\prime}, t_{\perp}\right) /\left(q_{f} B\right)\right] \operatorname{tr}_{D}\left[\tilde{S}_{f}\left(p_{\perp}, q_{\|}^{+}\right) \bar{\Gamma}^{C} \gamma_{\mu} \tilde{S}_{f}\left(p_{\perp}^{\prime}, q_{\|}^{-}\right) \gamma_{5}\right], \\
& F_{\mu}^{C,(\mathrm{IIII})}(t)=-8 \pi^{2} \sigma N_{C} \sum_{f=u, d} \frac{1}{B_{f}^{2}} \int_{q_{\|} r_{\|} q_{\perp} r_{\perp} p_{\perp} p_{\perp}^{\prime}} g\left(q^{2}\right) \exp \left[i 2 \varphi\left(q_{\perp}, p_{\perp}, p_{\perp}^{\prime}, t_{\perp}\right) /\left(q_{f} B\right)\right] \\
& \times \operatorname{tr}_{D}\left[\tilde{S}_{f}\left(p_{\perp}, q_{\|}^{+}\right) \Gamma^{C} \tilde{S}_{f}\left(p_{\perp}^{\prime}, q_{\|}^{-}\right) \gamma_{5}\right]\left\{g\left(\left(p_{\perp}+p_{\perp}^{\prime}-q_{\perp}-r_{\perp} / 2\right)^{2}+\left(q_{\|}-r_{\|} / 2\right)^{2}\right)\right. \\
&\left.-g\left(\left(p_{\perp}+p_{\perp}^{\prime}-q_{\perp}-r_{\perp} / 2+t_{\perp} / 2\right)^{2}+\left(q_{\|}-r_{\|} / 2+t_{\|} / 2\right)^{2}\right)\right\} h_{\mu}(r, t-r),
\end{aligned}
$$

where

$h_{\mu}(q, t-q)=\int d^{4} z \exp [-i(t-q) z] \int_{0}^{z} d \ell_{\mu} \exp [i t \ell]$

and

$$
\begin{aligned}
\varphi\left(q_{\perp}, p_{\perp}, p_{\perp}^{\prime}, t_{\perp}\right)= & p_{2}\left(q_{1}-t_{1} / 2\right)-p_{2}^{\prime}\left(q_{1}+t_{1} / 2\right) \\
& -q_{1} t_{2}-p_{2} p_{1}^{\prime}-(1 \leftrightarrow 2) .
\end{aligned}
$$

As in the case of the calculation of the $\pi^{0}$ mass and wave function renormalization, the integrals over transverse momenta can be performed analytically after LaguerreFourier transforming the nonlocal form factor functions. The steps to be followed in each case are outlined in
Appendix B. In what follows we just quote the results of this rather lengthy calculation. The form factors are evaluated at the pion pole, i.e. $t_{\|}^{2}=-m_{\pi^{0}}^{2}, t_{\perp}^{2}=0$.

The calculation of $f_{\pi^{0}}^{(A \|)}$ has been previously performed in Ref. [24], where the contributions from $F_{\mu}^{C,(\mathrm{I})}(t)$, $F_{\mu}^{C,(\mathrm{II})}(t)$ and $F_{\mu}^{C,(\mathrm{III})}(t)$ are quoted. Summing all three contributions one has [24]

$$
\begin{aligned}
\left.t_{\|} \cdot F_{\|}^{A}(t)\right|_{t_{\perp}^{2}=0}= & i \frac{N_{C}}{\pi} \sum_{f=u, d} B_{f} \sum_{k=0}^{\infty} \int_{q_{\|}} \sum_{\lambda= \pm} g_{k, q_{\|}}^{\lambda, f}\left(M_{k, q_{\|}}^{\lambda, f} F_{k k, q_{\|}^{+} q_{\|}^{-}}^{\lambda, f(A B)}\right. \\
& \left.+2 k B_{f} M_{k, q_{\|}}^{-\lambda, f} F_{k k, q_{\|}^{+} q_{\|}^{-}}^{\lambda, f(C D)}-\hat{A}_{k, q_{\|}}^{\lambda, f}\right)
\end{aligned}
$$

Taking into account this result, and making use of Eq. (32) and the gap equation (29), one arrives at [24]

$$
f_{\pi^{0}}^{(A \|)}=-m_{c} Z_{\|}^{1 / 2} \frac{N_{C}}{\pi t_{\|}^{2}} \sum_{f=u, d} B_{f} \sum_{k=0}^{\infty} \int_{q_{\|}} \sum_{\lambda= \pm} g_{k, q_{\|}}^{\lambda, f}\left(F_{k k, q_{\|}^{+} q_{\|}^{-}}^{\lambda, f(A B)}+\left.2 k B_{f} F_{k k, q_{\|}^{+} q_{\|}^{-}}^{\lambda, f(C D)}\right|_{t_{\|}^{2}=-m_{\pi^{0}}^{2}}\right.
$$

In the case of $f_{\pi^{0}}^{(V)}$, it is seen that $F_{\mu}^{V, \text { (I) }}(t)$ vanish identically, and the contribution from $F_{\mu}^{V \text {,(III) }}(t)$ is zero. From $F_{\mu}^{V, \text { (II) }}(t)$ one obtains 


$$
f_{\pi^{0}}^{(V \|)}=Z_{\|}^{1 / 2} \frac{N_{C}}{\pi} \sum_{f=u, d} B_{f} \sum_{k=0}^{\infty} \int_{q_{\|}} \frac{\left(q_{\|}^{+} \cdot t_{\|}\right)}{t_{\|}^{2}} \times\left.\sum_{\lambda= \pm} \lambda g_{k, q_{\|}}^{\lambda, f}\left(\hat{A}_{k, q_{\|}^{-}}^{\lambda, f} \hat{B}_{k, q_{\|}^{+}}^{\lambda, f}-2 k B_{f} \hat{C}_{k, q_{\|}^{-}}^{\lambda, f} \hat{D}_{k, q_{\|}^{+}}^{\lambda, f}\right)\right|_{t_{\|}^{2}=-m_{\pi^{0}}^{2}} .
$$

Finally, for $f_{\pi^{0}}^{(A \|)}$ the calculations sketched in Appendix B lead to

$$
\begin{aligned}
& \left.\frac{t_{\perp} \cdot F_{\perp}^{A,(\mathrm{I})}(t)}{t_{\perp}^{2}}\right|_{t_{\perp}^{2}=0}=i \frac{N_{C}}{4 \pi} \sum_{f=u, d} B_{f} \sum_{k=0}^{\infty} \sum_{\lambda= \pm} \int_{0}^{1} d \beta \beta \int_{q_{\|}} \hat{A}_{k, q_{\|}}^{\lambda, f}\left\{2 g_{k, q_{\beta \|}^{\prime}}^{\lambda, f}\right. \\
& \left.+B_{f}\left[\left(k_{\lambda}+1\right)\left(g_{k+1, q_{\beta \|}^{+}}^{\prime \prime \lambda, f}+g_{k, q_{\beta \|}^{+}}^{\prime \prime \lambda, f}\right)+k_{\lambda}\left(g_{k, q_{\beta \|}^{+}}^{\prime \prime \lambda, f}+g_{k-1, q_{\beta \|}^{+}}^{\prime \prime \lambda, f}\right)\right]\right\} \text {, } \\
& \left.\frac{t_{\perp} \cdot F_{\perp}^{A,(\mathrm{II})}(t)}{t_{\perp}^{2}}\right|_{t_{\perp}^{2}=0}=i \frac{N_{C}}{2 \pi} \sum_{f=u, d} B_{f} s_{f} \sum_{k=0}^{\infty} \int_{q_{\|}} \sum_{\lambda= \pm} \lambda\left[2 k g_{k, q_{\|}}^{\lambda, f} H_{k k, q_{\|}^{+} q_{\|}^{-}}^{\lambda, f}-k_{\lambda}\left(g_{k-1, q_{\|}}^{\lambda, f}+g_{k, q_{\|}}^{\lambda, f}\right) H_{k_{-\lambda} k_{\lambda}, q_{\|}^{+} q_{\|}^{-}}^{\lambda, f},\right. \\
& \left.\frac{t_{\perp} \cdot F_{\perp}^{A,(\mathrm{III})}(t)}{t_{\perp}^{2}}\right|_{t_{\perp}^{2}=0}=-i \frac{\bar{\sigma} N_{C}}{4 \pi} \sum_{f=u, d} B_{f} \sum_{k=0}^{\infty} \int_{q_{\|}} \sum_{\lambda= \pm}\left\{\left(g_{k, q_{\|}}^{\lambda, f} F_{k k, q_{\|}^{+} q_{\|}^{-}}^{\lambda, f(A B)}+2 k B_{f} g_{k, q_{\|}}^{-\lambda, f} F_{k k, q_{\|}^{+} q_{\|}^{-}}^{\lambda, f(C D)}\right)\right. \\
& \times \int_{0}^{1} d \beta \beta\left[2 g_{k, q_{\beta \|}^{+}}^{\prime \lambda, f}+B_{f}\left[\left(k_{\lambda}+1\right)\left(g_{k+1, q_{\beta \|}^{+}}^{\prime \prime \lambda, f}+g_{k, q_{\beta \|}^{+}}^{\prime \prime \lambda, f}\right)+k_{\lambda}\left(g_{k, q_{\beta \|}^{+}}^{\prime \prime \lambda, f}+g_{k-1, q_{\beta \|}^{+}}^{\prime \prime \lambda, f}\right)\right]\right] \\
& -\frac{k_{\lambda}}{2}\left[( g _ { k - 1 , q _ { \| } } ^ { \lambda , f } + g _ { k , q _ { \| } } ^ { \lambda , f } ) \left(F_{k-1 k, q_{\|}^{+} q_{\|}^{-}}^{\lambda, f(A B)}-F_{k k-1, q_{\|}^{+} q_{\|}^{-}}^{\lambda, f(A B)}+2 k_{-\lambda} B_{f}\right.\right. \\
& \left.\left.\times\left(g_{k-1, q_{\|}}^{-\lambda, f}+g_{k, q_{\|}}^{-\lambda, f}\right)\left(F_{k-1 k, q_{\|}^{+} q_{\|}^{-}}^{+, f(C D)}-F_{k k-1, q_{\|}^{+} q_{\|}^{-}}^{+, f(C D)}\right)\right] \int_{0}^{1} d \beta\left(g_{k, q_{\beta \|}^{+}}^{\lambda, f}+g_{k-1, q_{\beta \|}^{+}}^{\lambda, f}\right)\right\},
\end{aligned}
$$

where $g_{k, q_{\|}}^{\prime \lambda, f}, g_{k, q_{\|}}^{\prime \prime \lambda, f}$ indicate derivations with respect to $q_{\|}^{2}$, and we have defined

$$
q_{\beta \|}^{+}=q_{\|}+\beta t_{\|} / 2, H_{k k^{\prime}, q_{\|}^{+} q_{\|}^{-}}^{\lambda, f}=\hat{A}_{k, q_{\|}^{+}}^{\lambda, f} \hat{C}_{k^{\prime}, q_{\|}^{-}}^{\lambda, f}-\left(q_{\|}^{+} \cdot q_{\|}^{-}\right) \hat{B}_{k, q_{\|}^{+}}^{\lambda, f} \hat{D}_{k^{\prime}, q_{\|}^{-}}^{\lambda, f}
$$

Summing these three contributions, and using the relation

$$
\left(g_{k+1, q_{\|}}^{\lambda, f}+g_{k, q_{\|}}^{\lambda, f}\right) B_{f}=g_{k+1, q_{\|}}^{\lambda, f}-g_{k, q_{\|}}^{\lambda, f}
$$

(which arises from the properties of Laguerre polynomials), we arrive at a final expression for $f_{\pi^{0}}^{(A \perp)}$, given by

$$
\begin{aligned}
& f_{\pi^{0}}^{(A \perp)}=Z_{\|}^{1 / 2} \frac{N_{C}}{4 \pi} \sum_{f=u, d} \sum_{k=0}^{\infty} \int_{q_{\|}} \sum_{\lambda= \pm}\left\{\left[-\hat{A}_{k, q_{\|}}^{\lambda, f}+\bar{\sigma}\left(g_{k, q_{\|}}^{\lambda, f} F_{k k, q_{\|}^{+} q_{\|}^{-}}^{\lambda, f(A B)}+2 k B_{f} g_{k, q_{\|}}^{-\lambda, f} F_{k k, q_{\|}^{+} q_{\|}^{-}}^{\lambda, f(C D)}\right)\right]\right. \\
& \times \int_{0}^{1} d \beta \beta\left[\left(k_{\lambda}+1\right)\left(g_{k+1, q_{\beta \|}^{+}}^{\lambda, f}-g_{k, q_{\beta \|}^{+}}^{\lambda, f}\right)-k_{\lambda}\left(g_{k, q_{\beta \|}^{+}}^{\lambda, f}-g_{k-1, q_{\beta \|}^{+}}^{\lambda, f}\right)\right] \\
& -2 B_{f} s_{f} \lambda\left[2 k g_{k, q_{\|}}^{\lambda, f} H_{k k, q_{\|}^{+} q_{\|}^{-}}^{\lambda, f}-k_{\lambda}\left(g_{k-1, q_{\|}}^{\lambda, f}+g_{k, q_{\|}}^{\lambda, f}\right) H_{k_{-\lambda} k_{\lambda}, q_{\|}^{+} q_{\|}^{-}}^{\lambda, f}\right] \\
& -\bar{\sigma} \frac{k_{\lambda}}{2}\left[\left(g_{k-1, q_{\|}}^{\lambda, f}+g_{k, q_{\|}}^{\lambda, f}\right)\left(F_{k-1 k, q_{\|}^{+} q_{\|}^{-}}^{\lambda, f(A B)}-F_{k k-1, q_{\|}^{+} q_{\|}^{-}}^{\lambda, f(A B)}\right)+2 k_{-\lambda} B_{f}\right. \\
& \left.\times\left(g_{k-1, q_{\|}}^{-\lambda, f}+g_{k, q_{\|}}^{-\lambda, f}\right)\left(F_{k-1 k, q_{\|}^{+} q_{\|}^{-}}^{\lambda, f(C D)}-F_{k k-1, q_{\|}^{+} q_{\|}^{-}}^{\lambda, f(C D)}\right) \int_{0}^{1} d \beta\left(g_{k, q_{\beta \|}^{+}}^{\lambda, f}-g_{k-1, q_{\beta \|}^{+}}^{\lambda, f}\right)\right\}\left.\right|_{t_{\|}^{2}=-m_{\pi^{2}}^{2}} .
\end{aligned}
$$




\section{Chiral relations}

It is interesting to study the relations involving form factors and renormalization constants in the chiral limit, $m_{c} \rightarrow 0$. First, taking into account the expression in Eq. (40), the gap equation (29) and the relation

$g_{k, q_{\|}}^{\lambda, f} F_{k k, q_{\|} q_{\|}}^{\lambda, f(A B)}+2 k B_{f} g_{k, q_{\|}}^{-\lambda, f} F_{k k, q_{\|} q_{\|}}^{\lambda, f(C D)}=\frac{1}{\bar{\sigma}}\left(\hat{A}_{k, q_{\|}}^{\lambda, f}-m_{c} \hat{B}_{k, q_{\|}}^{\lambda, f}\right)$,

it is seen that

$F(0,0)=-\frac{1}{G}+\frac{m_{c}}{\bar{\sigma}} \frac{N_{C}}{\pi} \sum_{f=u, d} B_{f} \sum_{k=0}^{\infty} \int_{q_{\|}} \sum_{\lambda= \pm} g_{k, q_{\|}}^{\lambda, f} \hat{B}_{k, q_{\|}}^{\lambda, f}$.

Thus, in the limit $m_{c} \rightarrow 0$, the second term on the rhs vanishes and from Eq. (32) one obtains $m_{\pi^{0}}=0$, as expected.

The validity of the Goldberger-Treiman relation

$$
f_{\pi^{0}, 0}^{(A \|)}=Z_{\|, 0}^{-1 / 2} \bar{\sigma}_{0}
$$

and the Gell-Mann-Oakes-Renner relation

$$
m_{c}\langle\bar{u} u+\bar{d} d\rangle_{0}=-m_{\pi^{0}}^{2} f_{\pi^{0}, 0}^{(A \|) 2}
$$

in the presence of the external magnetic field have been shown in Ref. [24]. In these equations, subindices 0 indicate that the quantities have to be evaluated in the chiral limit. Now let us take into account the expression for $f_{\pi^{0}}^{(A \perp)}$ in Eq. (64). For $m_{c}=0, m_{\pi^{0}}=0$, it is seen that the first term in curly brackets is zero owing to Eq. (65), while the last two terms also vanish since $F_{k k^{\prime}, q_{\|} q_{\|}}^{\lambda, f(X Y)}$ is symmetric under the exchange between $k$ and $k^{\prime}$. Moreover, it is easy to see that

$$
H_{k k, q_{\|} q_{\|}}^{\lambda, f}=\frac{M_{k, q_{\|}}^{-\lambda, f}}{\Delta_{k, q_{\|}}^{f}},
$$

from which the piece proportional to $H_{k k, q_{\|} q_{\|}}^{\lambda, f}$ also vanishes. One gets in this way

$$
f_{\pi^{0}, 0}^{(A \perp)}=Z_{\|, 0}^{1 / 2} \frac{N_{C}}{2 \pi} \sum_{f=u, d} B_{f} s_{f} \sum_{k=0}^{\infty} \int_{q_{\|}} \sum_{\lambda= \pm} \lambda k_{\lambda}\left(g_{k-1, q_{\|}, 0}^{\lambda, f}+g_{k, q_{\|}, 0}^{\lambda, f}\right) H_{k_{-\lambda} k_{\lambda}, q_{\|} q_{\|}, 0}^{\lambda, f} .
$$

On the other hand, from Eqs. (41) and (65) it is seen that in the chiral limit one has

$$
\begin{aligned}
Z_{\perp, 0}^{-1}= & \frac{N_{C}}{4 \pi} \sum_{f=u, d} \sum_{k=0}^{\infty} \int_{q_{\|}} \sum_{\lambda= \pm} k_{\lambda}\left(g_{k_{\lambda}, q_{\|}, 0}^{\lambda, f}+g_{k_{-\lambda}, q_{\|}, 0}^{\lambda, f}\right)\left[\frac{1}{\bar{\sigma}_{0}}\left(\hat{A}_{k_{\lambda}, q_{\|}, 0}^{\lambda, f}+\hat{A}_{k_{-\lambda}, q_{\|}, 0}^{\lambda, f}\right)\right. \\
& \left.-\left(g_{k_{\lambda}, q_{\|}, 0}^{\lambda, f}+g_{k_{-\lambda}, q_{\|}, 0}^{\lambda, f}\right) F_{k_{\lambda} k_{-\lambda}, q_{\|} q_{\|}, 0}^{\lambda, f(A B)}-2 k_{-\lambda} B_{f}\left(g_{k_{\lambda}, q_{\|}, 0}^{-\lambda, f}+g_{k_{-\lambda}, q_{\|}, 0}^{-\lambda, f}\right) F_{k_{\lambda} k_{-\lambda}, q_{\|} q_{\|}, 0}^{\lambda, f(C D)}\right] .
\end{aligned}
$$

After some algebra, it can be shown that the factor in square brackets is equal to $2 B_{f}\left(k_{\lambda}-k_{-\lambda}\right) H_{k_{-\lambda} k_{\lambda}, q_{\|} q_{\|}, 0}^{h_{0}} / \bar{\sigma}_{0}$. Since $k_{\lambda}-k_{-\lambda}=s_{f} \lambda$, by comparing with Eq. (70) one finally gets

$$
f_{\pi^{0}, 0}^{(A \perp)}=Z_{\|, 0}^{1 / 2} Z_{\perp, 0}^{-1} \bar{\sigma}_{0}
$$

Thus, taking into account Eq. (67), one has

$$
\frac{f_{\pi^{0}, 0}^{(A \perp)}}{f_{\pi^{0}, 0}^{(A \|)}}=\frac{Z_{\|, 0}}{Z_{\perp, 0}}=u_{\pi^{0}, 0}^{2} .
$$

This result has been also found in the framework of the local NJL model in Ref. [48] and (using a different notation) in Ref. [10], where it is obtained from a modified PCAC relation.

\section{FINITE TEMPERATURE}

In this section we extend the previous analysis to a system at finite temperature using the standard Matsubara formalism. To describe the confinement/deconfinement transitions we include a coupling between the fermions and the Polyakov loop (PL), assuming that the quarks move on a uniform background color field. This type of interactions has been previously considered in nonlocal models [35-38,41], as well as in the local Polyakov-Nambu-JonaLasinio (PNJL) model [49-52] and in Polyakov-quarkmeson models $[53,54]$. The background field is given by $\phi=i g \delta_{\mu 0} G_{a}^{\mu} \lambda^{a} / 2$, where $G_{a}^{\mu}$ are the $\mathrm{SU}(3)$ color gauge 
fields. Working in the so-called Polyakov gauge the matrix $\phi$ is given a diagonal representation $\phi=\phi_{3} \lambda_{3}+\phi_{8} \lambda_{8}$, and the traced Polyakov loop $\Phi=\frac{1}{3} \operatorname{Tr} \exp (i \phi / T)$ can be taken as an order parameter of the confinement/deconfinement transitions. Since at mean field $\Phi$ is expected to be real owing to charge conjugation symmetry, one has $\phi_{8}=0$ and $\Phi=\left[1+2 \cos \left(\phi_{3} / T\right)\right] / 3$ [52]. In addition, we include a Polyakov-loop potential $\mathcal{U}(\Phi, T)$ that accounts for effective gauge field self-interactions. The mean field grand canonical thermodynamic potential of the system per unit volume under the external magnetic field is given by [41]

$$
\begin{aligned}
\Omega_{B, T}^{\mathrm{MFA}}= & \frac{\bar{\sigma}^{2}}{2 G}-T \sum_{f=u, d} \frac{\left|q_{f} B\right|}{2 \pi} \sum_{n=-\infty}^{\infty} \sum_{c} \\
& \times \int \frac{d p_{3}}{2 \pi}\left[\ln \left(p_{\| n c}^{2}+M_{0, p_{\| n c}}^{s_{f}, f}{ }^{2}\right)\right. \\
& \left.+\sum_{k=1}^{\infty} \ln \left(\Delta_{k, p_{\| n c}}^{f}\right)\right]+\mathcal{U}(\Phi, T),
\end{aligned}
$$

where $\Delta_{k, p_{\| n c}}^{f}$ is the function in Eq. (17), and we have defined $\vec{p}_{\| n c}=\left(p_{3}, p_{4 n c}\right)$, with $p_{4 n c}=(2 n+1) \pi T+\phi_{c}$. The sum over color indices runs over $c=r, g, b$, and color background fields are $\left(\phi_{r}, \phi_{g}, \phi_{b}\right)=\left(\phi_{3},-\phi_{3}, 0\right)$.

Since $\Omega_{B, T}^{\mathrm{MFA}}$ is divergent, it has to be properly regularized. We take the prescription followed in Ref. [41], in which one subtracts to $\Omega_{B, T}^{\mathrm{MFA}}$ the thermodynamic potential of a free fermion gas, and then adds it in a regularized form. The regularized potential is given by

$$
\Omega_{B, T}^{\mathrm{MFA}, \text { reg }}=\Omega_{B, T}^{\mathrm{MFA}}-\Omega_{B, T}^{\mathrm{free}}+\Omega_{B, T}^{\mathrm{free}, \text { reg }} .
$$

In fact, the "free" piece keeps the interaction with the magnetic field and the PL. The explicit expression of $\Omega_{B, T}^{\text {free,reg }}$, for which the Matsubara sums can be performed analytically, can be found in Ref. [41].

The form of the PL potential is an additional input of the model. In this work we take a widely used polynomial form based on a Ginzburg-Landau ansatz, namely [51,55]

$$
\frac{\mathcal{U}(\Phi, T)}{T^{4}}=-\frac{b_{2}(T)}{2} \Phi^{2}-\frac{b_{3}}{3} \Phi^{3}+\frac{b_{4}}{4} \Phi^{4},
$$

where

$$
b_{2}(T)=a_{0}+a_{1}\left(\frac{T_{0}}{T}\right)+a_{2}\left(\frac{T_{0}}{T}\right)^{2}+a_{3}\left(\frac{T_{0}}{T}\right)^{3} .
$$

The numerical values for the parameters $a_{i}$ and $b_{i}$, which can be obtained by a fit to pure gauge lattice QCD results, can be found in Ref. [51]. This potential leads to a firstorder phase transition at $T_{0}$, which becomes a further parameter of the model. In the absence of dynamical quarks, form lattice QCD one would expect a deconfinement temperature of about $T_{0}=270 \mathrm{MeV}$. However, it has been argued that in the presence of light dynamical quarks this parameter should be reduced. For definiteness we will take $T_{0} \simeq 200 \mathrm{MeV}$, as suggested in Refs. [53,54].

The values of $\bar{\sigma}$ and $\phi_{3}$ at the mean field level can be found by minimizing the regularized thermodynamic potential, i.e. by solving the coupled equations:

$$
\frac{\partial \Omega_{B, T}^{\mathrm{MFA}, \mathrm{reg}}}{\partial \bar{\sigma}}=0, \quad \frac{\partial \Omega_{B, T}^{\mathrm{MFA}, \mathrm{reg}}}{\partial \phi_{3}}=0 .
$$

Finite temperature meson masses and decay constants can be then calculated from Eqs. (32), (40), (57), (58) and (64), following the prescription

$N_{C} \int_{q_{\|}} F\left(q_{\|}, t_{\|}\right) \rightarrow T \sum_{n=-\infty}^{\infty} \sum_{c} \int \frac{d q_{3}}{2 \pi} F\left(q_{\| n c}, t_{\|}\right)$

and taking the external momentum $\vec{t}_{\|}=\left(\operatorname{im}_{\pi^{0}}(T), 0\right)$. Notice that these mass values correspond to spatial "screening masses" for the zeroth bosonic Matsubara mode $\left(t_{4}=0\right)$. The reciprocals $m_{\pi^{0}}(T)^{-1}$ can be understood as the persistence lengths of this mode, in equilibrium with the heat bath.

\section{NUMERICAL RESULTS}

To obtain numerical predictions for the behavior of the quantities defined in the previous sections, it is necessary to specify the model parameters and the shape of the nonlocal form factor $g\left(p^{2}\right)$. We consider here the often-used Gaussian function

$$
g\left(p^{2}\right)=\exp \left(-p^{2} / \Lambda^{2}\right) .
$$

In general, the form factor introduces an energy scale $\Lambda$ that represents an effective momentum cutoff. This constant has to be taken as a free parameter of the model, together with the current quark mass $m_{c}$ and the coupling constant $G$ in the effective Lagrangian. In the particular case of the Gaussian form factor one has the advantage that the integral in Eq. (20) can be performed analytically, which leads to a dramatic reduction of the computer time needed for numerical calculations of meson masses and form factors.

As in Refs. [40,41], we fix the free parameters by requiring the model to reproduce the empirical values (for vanishing external field) of the pion mass and decay constant, as well as some phenomenologically adequate values of the quark condensate $\langle\bar{f} f\rangle(f=u, d)$. Some parameter sets, corresponding to different values of the condensate, can be found in Ref. [41]. Here we take $m_{c}=6.5 \mathrm{MeV}, \Lambda=678 \mathrm{MeV}$ and $G \Lambda^{2}=23.66$, which lead to $\langle\bar{f} f\rangle=(-230 \mathrm{MeV})^{3}$. This will be called set I. As shown in Ref. [41], for this parametrization the behavior of 
quark condensates with the magnetic field, at zero temperature, are found to be in very good agreement with lattice QCD results. These parameters have also been used in the previous calculation of $m_{\pi^{0}}$ and $f_{\pi^{0}}^{(A \|)}$ for nonzero $B$ carried out in Ref. [24]. In order to test the sensitivity of our results to the parameters we also consider two alternative sets, which correspond to quark condensates $\langle\bar{f} f\rangle=(-220 \mathrm{MeV})^{3}$ and $\langle\bar{f} f\rangle=(-240 \mathrm{MeV})^{3}$ in vacuum. The latter are denoted as sets II and III, respectively.
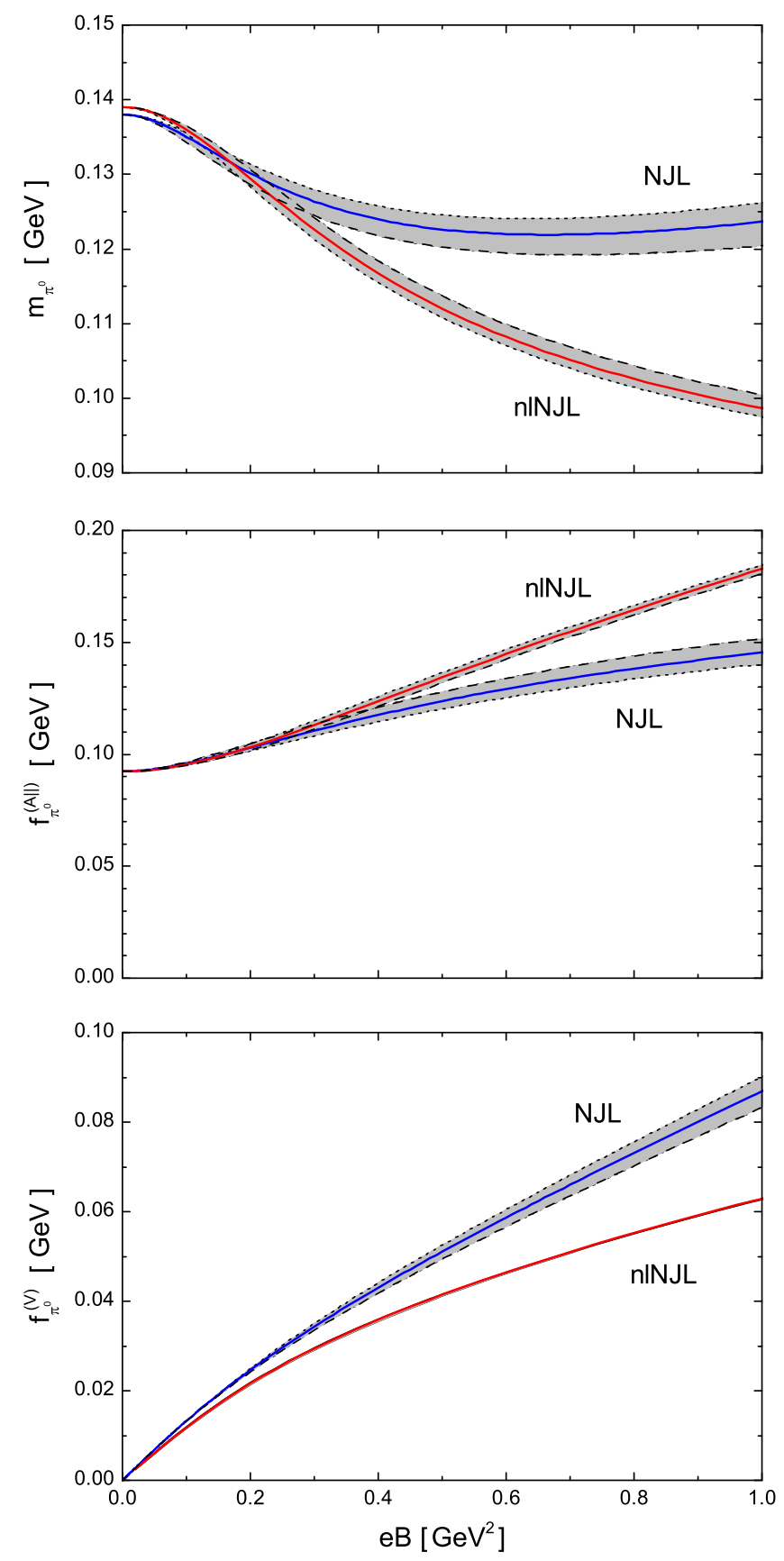

In Fig. 1 we show our numerical results for various quantities associated with the neutral pion at zero temperature, as functions of $e B$. Solid red lines denote the results from set I, while the limits of the corresponding gray bands are determined by the results from set II (dashed lines) and set III (dotted lines). For comparison we also include in the figure the numerical results obtained within the local NJL model, quoted in Ref. [48]. Solid blue lines correspond to a parametrization leading to a constituent quark mass $M=350 \mathrm{MeV}$ (for $B=0$ ), while the limits of the gray
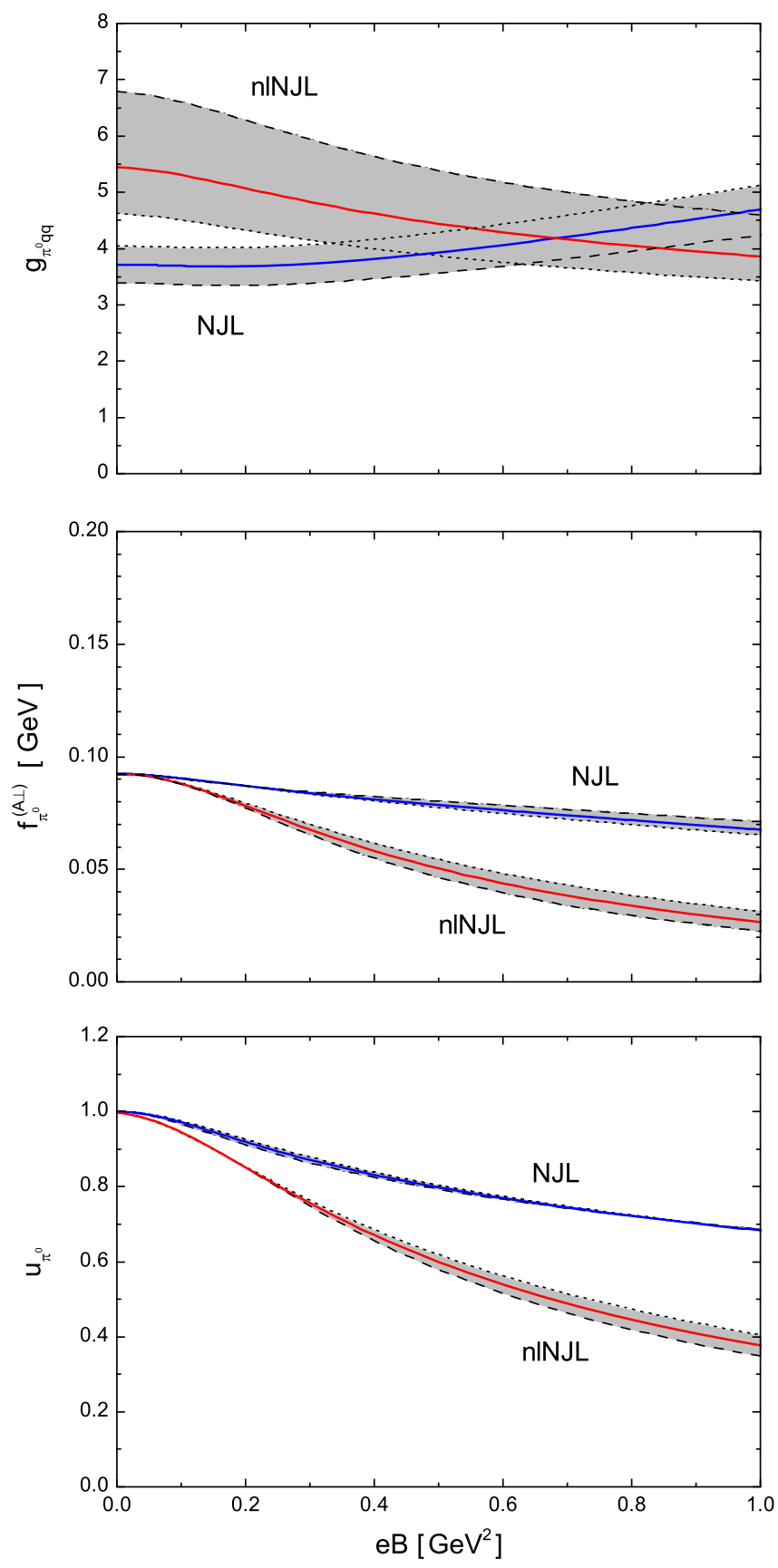

FIG. 1. Neutral pion properties as functions of $e B$. Solid red lines correspond to set I, while the limits of the gray bands correspond to set II (dashed lines) and set III (dotted lines). Solid blue lines and associated gray bands correspond to local NJL results (see text). 
bands correspond to $M=320 \mathrm{MeV}$ (dashed lines) and $M=$ $380 \mathrm{MeV}$ (dotted lines). The values of the quark-antiquark condensates for these parametrizations of the NJL model are $\langle\bar{q} q\rangle \simeq(-243 \mathrm{MeV})^{3},(-236 \mathrm{MeV})^{3}$ and $(-250 \mathrm{MeV})^{3}$, respectively. It should be noted that the results for the pion mass and the $f_{\pi^{0}}^{(A \|)}$ form factor have been previously obtained in Ref. [24].

From the graphs in Fig. 1 it can be said that in general our results do not show a large dependence with the model parametrization. As shown in the upper left panel in Fig. 1, the dependence of the $\pi^{0}$ mass with the external field for the nonlocal effective model is significantly stronger than in the case of the local NJL approach. In the upper right panel of the figure we plot the curves for the effective coupling constant $g_{\pi^{0} q q}$, which shows different behaviors for nINJL and NJL models. In the left and right central panels of the figure we quote the curves corresponding to the axial form factors. Notice that for $B=0$ one has spacial rotation symmetry and both $f_{\pi^{0}}^{(A \|)}$ and $f_{\pi^{0}}^{(A \perp)}$ reduce to the usual $\pi^{0}$ decay constant [see Eqs. (43)]. As the magnetic field increases, $f_{\pi^{0}}^{(A \|)}$ gets enhanced and $f_{\pi^{0}}^{(A \perp)}$ gets reduced. This is in qualitative agreement with the results for the local NJL model, although for the latter the $B$ dependence is noticeably milder The lower left panel shows the behavior of the vector form factor $f_{\pi^{0}}^{(V)}$ as a function of $e B$. This form factor is zero at vanishing external field and shows a monotonic growth with $e B$, with little dependence on the parametrization. In this case the growth is shown to be somewhat steeper for the local NJL model. Finally, in the lower right panel we quote the curves for directional refraction index $u_{\pi^{0}}$, which is found to get reduced for increasing external field.

The results for the above discussed quantities, together with those obtained for $\langle\bar{q} q\rangle$ (for the analysis of condensates and related quantities, see Refs. [40,41]), allow us to check the validity of the chiral relations in Eqs. (68) and (73). They are found to be satisfied within 5\% and $0.2 \%$ accuracy, respectively, for $e B$ up to $1.5 \mathrm{GeV}^{2}$ (for definiteness, we have considered parameter set I). In particular, the opposite behavior of $f_{\pi^{0}}^{(A \|)}$ and $f_{\pi^{0}}^{(A \perp)}$ with the magnetic field can be understood from Eq. (73), taking into account that $u_{\pi^{0}}$ becomes significantly reduced for increasing $B$. In the NJL, it has also been shown that the relation $f_{\pi^{0}}^{(V)}=$ $e B /\left(8 \pi^{2} f_{\pi^{0}}^{(A \|)}\right)$ holds in the chiral limit [48]. We have checked this relation numerically in the nlNJL model, finding that it remains only approximately valid (that is, within a $15 \%$ accuracy) for the chosen range of $e B$.

We turn now to our results for a system at finite temperature. As expected, at some critical temperature $T_{c}(B)$ the system undergoes a crossover transition in which chiral symmetry is partially restored. Moreover, as shown in Refs. [40,41], this model leads to inverse magnetic catalysis, in the sense that $T_{c}$ is found to be a decreasing function of $B$. This is in agreement with lattice QCD results [43]. It has been also shown that there is a very small splitting between chiral restoration and deconfinement transition temperatures, the latter being defined according to the behavior of the Polyakov loop $\Phi$ (see e.g. Ref. [41] for details).

Regarding the quantities we are interested in here, in Fig. 2 we show the behavior of the $\pi^{0}$ and $\sigma$ meson masses (upper panel), and the normalized $\pi^{0}$ axial and vector decay form factors (lower panel) as functions of the temperature, for three representative values of the external magnetic field, namely $e B=0, e B=0.6 \mathrm{GeV}^{2}$ and $e B=1 \mathrm{GeV}^{2}$. The curves correspond to parameter set I and a polynomial Polyakov loop potential, as discussed in Sec. III. It can be seen that for nonzero $B$ the masses show a similar qualitative behavior with $T$ as in the $B=0$ case. The $\pi^{0}$ mass remains approximately constant up to the critical temperature, and $\pi^{0}$ and $\sigma$ masses match above $T_{c}$, as expected from chiral symmetry. For large temperatures it is seen that the masses get steadily increased, the growth being dominated by pure thermal effects. As stated, the critical temperature gets lower for increasing $B$. The critical

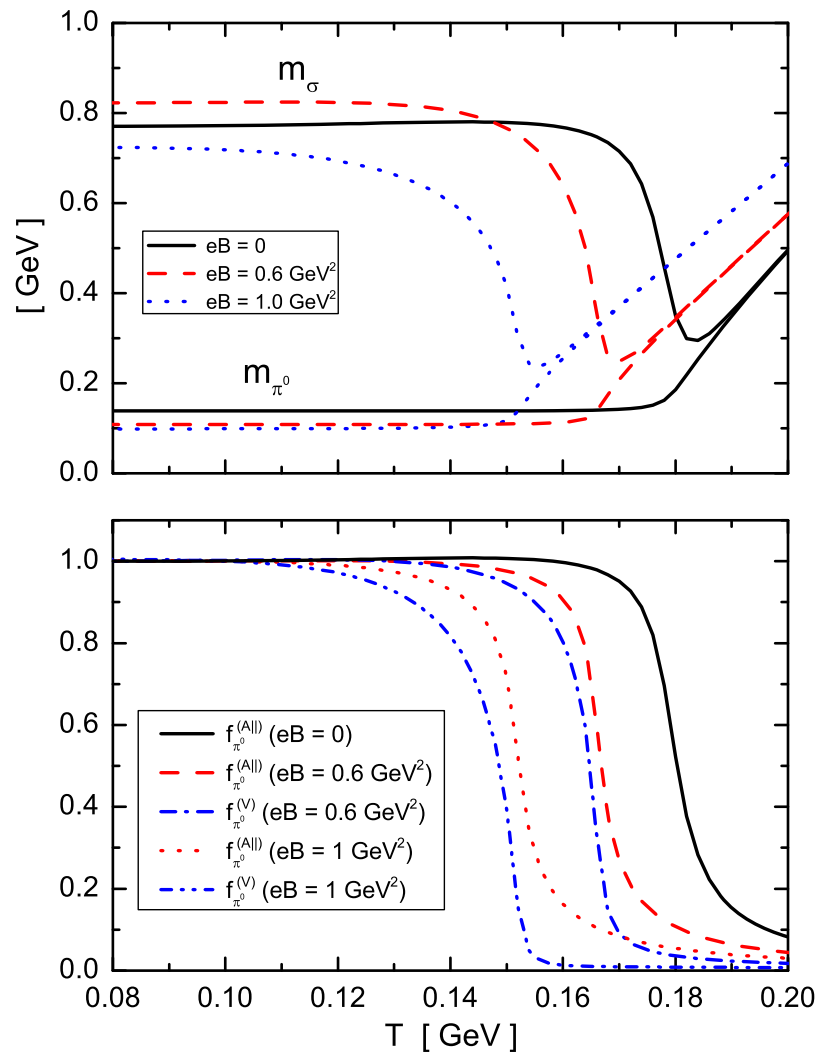

FIG. 2. $\pi^{0}$ and $\sigma$ meson masses (upper panel) and normalized $\pi^{0}$ decay form factors (lower panel) as functions of the temperature. Solid lines correspond to $e B=0$, dashed and dash-dotted lines to $e B=0.6 \mathrm{GeV}^{2}$, and dotted and dash-dot-dotted lines to $e B=1 \mathrm{GeV}^{2}$. 
temperatures for the chosen values $e B=0,0.6 \mathrm{GeV}^{2}$ and $1 \mathrm{GeV}^{2}$ are found to be $T_{c} \sim 180,165$ and $150 \mathrm{MeV}$, respectively [41]. In the case of the form factors, the curves for $f_{\pi^{0}}^{(A \|)}$ show a drop at the critical temperatures, exhibiting once again a qualitatively similar behavior for zero and nonzero external magnetic field. The curves for $f_{\pi^{0}}^{(A \perp)}$, normalized to $f_{\pi^{0}}^{(A \perp)}(T=0)$, differ from those of $f_{\pi^{0}}^{(A \|)}$ by less than about $5 \%$. For clarity they have not been included in the figure. Finally, the vector form factor $f_{\pi^{0}}^{(V)}$ also shows a drop at $T \sim T_{c}(B)$. The transition in this case is somewhat steeper than for $f_{\pi^{0}}^{(A \|)}$, and occurs at a slightly lower temperature. We recall that, at any temperature, $f_{\pi^{0}}^{(V)}$ is zero for vanishing external field.

For completeness, in Figs. 3 and 4 we show the behavior of meson properties as functions of $e B$ for three representative values of the temperature, namely $T=0,165$ and $180 \mathrm{MeV}$. The results for $T=0$, same as those previously shown in Fig. 1, are included just for comparison. The curves for $T=165 \mathrm{MeV}$ can be understood by looking at the results in Fig. 2, which show that this is the critical temperature that corresponds to $e B \simeq 0.6 \mathrm{GeV}^{2}$. Thus, the pion mass and form factors in Figs. 3 and 4 show approximately the same behavior as for $T=0$ up to

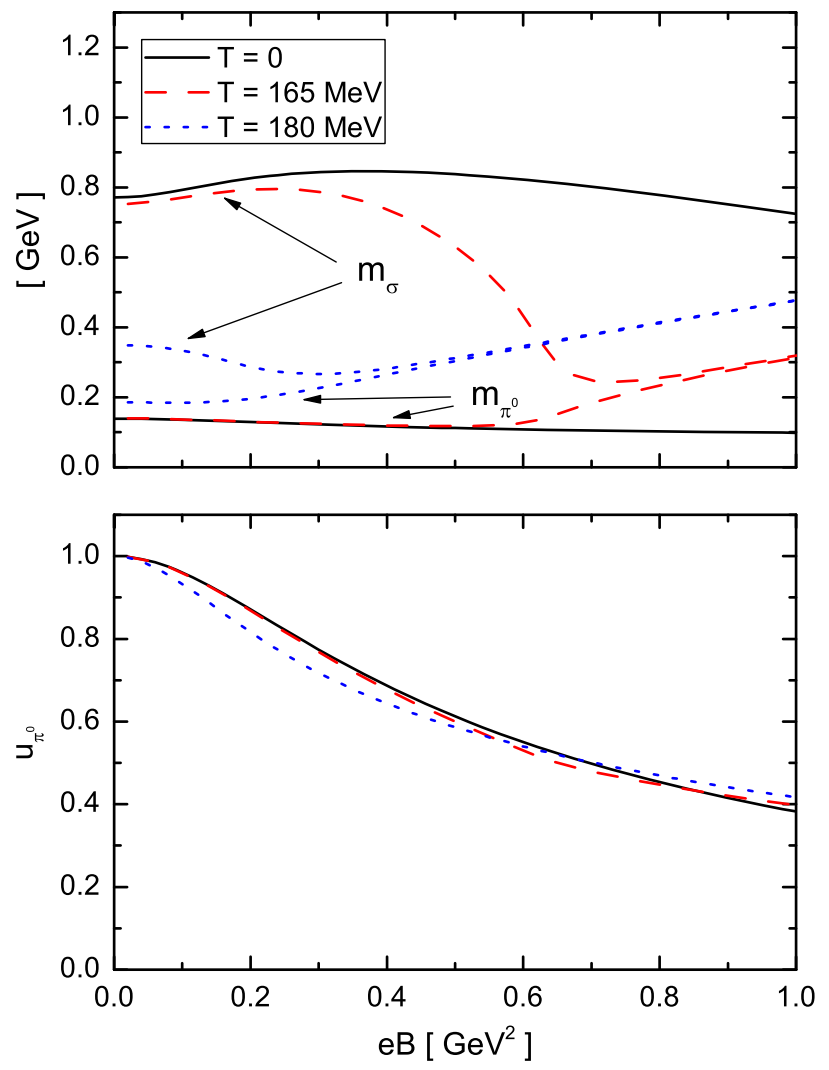

FIG. 3. $\pi^{0}$ and $\sigma$ meson masses (upper panel) and directional refraction index (lower panel) as functions of $e B$, for three representative values of the temperature.
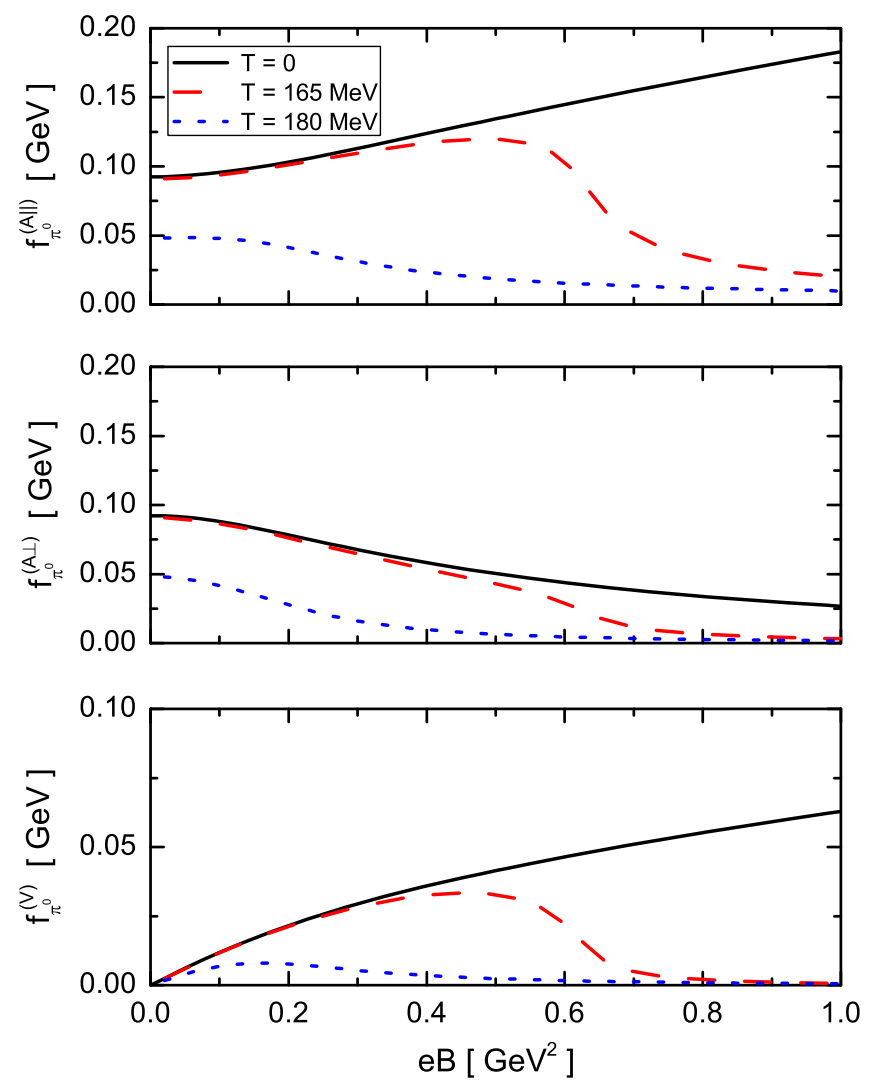

FIG. 4. From top to bottom, decay form factors $f_{\pi^{0}}^{(A \|)}, f_{\pi^{0}}^{(A \perp)}$ and $f_{\pi^{0}}^{(V)}$ as functions of $e B$, for three representative values of the temperature.

$e B \sim 0.5-0.6 \mathrm{GeV}^{2}$. Beyond these values, as expected from the results in Fig. 2, one finds an enhancement in the pion mass and a decrease in the axial and vector form factors. On the other hand, the curves for $T=180 \mathrm{MeV}$ are consistent with the fact that the chiral restoration transition occurs at approximately this temperature for vanishing magnetic field; the values of the pion mass and axial form factors are well separated from the $T=0$ values already at $B=0$. Finally, as shown in the lower panel of Fig. 3, the behavior of the directional refraction index $u_{\pi^{0}}$ is found to be basically independent of the temperature.

\section{SUMMARY AND CONCLUSIONS}

We have studied the behavior of neutral meson properties in the presence of a uniform static external magnetic field $B$, in the context of the so-called nINJL model. That is, a nonlocal effective approach based on the Nambu-JonaLasinio chiral quark model. Our analysis is a sort of extension of the work carried out in Ref. [24], where the pion mass and the decay form factor $f_{\pi^{0}}^{(A \|)}$, at zero temperature, were studied in this same framework. In the nlNJL model the effective couplings between quark-antiquark currents include nonlocal form factors that regularize 
ultraviolet divergences in quark loop integrals, and lead to a momentum-dependent effective mass in quark propagators. In order to obtain closed analytical expressions for meson polarization functions and pion decay constants in the presence of the external magnetic field, we have worked out a formalism that involves Ritus transforms of the Dirac fields.

We have first concentrated in the analysis at zero temperature of the form factors associated with pion-tovacuum matrix elements of the vector and axial vector hadronic currents. In agreement with the model independent analysis in Ref. [44], it is seen that for nonzero $B$ three nonvanishing independent form factors can be defined. Two of them, $f_{\pi^{0}}^{(A \|)}$ and $f_{\pi^{0}}^{(A \perp)}$, correspond to the axialvector current, and the other one, $f_{\pi^{0}}^{(V)}$, to the vector piece. We have also calculated the neutral pion directional refraction index, $u_{\pi^{0}}$, which in general is different from one. In addition, chiral relations are shown to be valid in the presence of the external field.

For the numerical calculations we have considered the case of Gaussian nonlocal form factors, choosing sets of model parameters that were previously found to reproduce the empirical values of the pion mass and decay constant at $B=0$, and lead to values of quark condensates at nonzero $B$ that are compatible with lattice QCD calculations. Taking into account external fields in the range from zero to $e B=1 \mathrm{GeV}^{2}$, from our numerical results it is noticed that all studied quantities show a strong dependence with $B$. As discussed in Sec. IV, in most cases this dependence is found to be significantly larger than that observed in the local NJL model [48]. On the other hand, it is seen that in general the results are rather stable under changes in the model parameters. It has been also verified that chiral relations remain approximately valid for the chosen parameter sets.

We have extended the calculations to finite temperature systems, including the couplings of fermions to the Polyakov loop and a PL polynomial potential that accounts for effective color gauge field self-interactions. As expected, it is seen that the system undergoes a phase transition corresponding to the restoration of $\mathrm{SU}(2)$ chiral symmetry. The model predicts the existence of inverse magnetic catalysis, leading to a decrease of the critical temperature $T_{c}$ with the magnetic field. Concerning the behavior of meson masses, it is seen that the $\pi^{0}$ mass remains approximately constant up to $T_{c}(B)$, while the $\sigma$ meson mass begins to drop earlier. Beyond the transition both masses become degenerate, as expected, and show a thermal growth for large $T$. Regarding the thermal behavior of the form factors, we observe that they remain approximately constant until temperatures close to $T_{c}(B)$ are reached, and then they show sudden drops. Finally, the directional refraction index $u_{\pi^{0}}$ is found to be basically independent of the temperature. To provide an alternative view, we have also included some graphs showing the behavior of the studied quantities as functions of the magnetic field, for some selected values of the temperature.

\section{ACKNOWLEDGMENTS}

This work has been supported in part by Consejo Nacional de Investigaciones Científicas y Técnicas and Agencia Nacional de Promoción Científica y Tecnológica (Argentina), under Grants No. PIP17-700 and No. PICT1703-0571, respectively, and by the National University of La Plata (Argentina), Project No. X824.

\section{APPENDIX A: DERIVATION OF THE EXPRESSION FOR $Z^{-1}$}

We outline here the derivation of the relation in Eq. (41), which can be obtained following a procedure similar to that described in the appendices of Ref. [24].

It is easy to see that the expression in Eq. (36) can be rearranged in the form

$$
F\left(t_{\perp}^{2}, t_{\|}^{2}\right)=-128 \pi^{2} N_{C} \sum_{f=u, d} \frac{1}{B_{f}^{2}} \sum_{k, k^{\prime}=0}^{\infty} \int_{q_{\|}}\left[\sum_{\lambda= \pm} F_{k k^{\prime}, q_{\|}^{+} q_{\|}^{-}}^{\lambda, f(A B)} I_{k k^{\prime}, q_{\|}}^{\lambda, f(0)}+F_{k k^{\prime}, q_{\|}^{+} q_{\|}^{-}}^{+, f(C D)} I_{k k^{\prime}, q_{\|}}^{f(1)}\right] .
$$

Taking the Laguerre-Fourier transforms of the nonlocal form factors given by Eq. (20), and changing the integration variables to dimensionless vectors $u=-\sqrt{\left(2 / B_{f}\right)} p_{\perp}, v=\sqrt{\left(2 / B_{f}\right)} p_{\perp}^{\prime}, w=\sqrt{\left(2 / B_{f}\right)}\left(p_{\perp}-q_{\perp}\right)$ and $r_{\perp}=\sqrt{\left(2 / B_{f}\right)} t_{\perp}$ in the plane perpendicular to $\vec{B}$, the integrals $I_{k k^{\prime}, q_{\|}}^{\lambda, f(0)}$ and $I_{k k^{\prime}, q_{\|}}^{f(1)}$ are given by

$$
\begin{aligned}
& I_{k k^{\prime}, q_{\|}}^{\lambda, f(0)}=\frac{B_{f}^{3}}{2}(-1)^{k+k^{\prime}} \sum_{m, m^{\prime}=0}^{\infty}(-1)^{m+m^{\prime}} g_{m, q_{\|}}^{\lambda, f} g_{m^{\prime}, q_{\|}}^{\lambda, f} K_{k k^{\prime} m m^{\prime}}^{\lambda, f(0)}, \\
& I_{k k^{\prime}, q_{\|}}^{f(1)}=2 B_{f}^{4}(-1)^{k+k^{\prime}} \sum_{m, m^{\prime}=0}^{\infty}(-1)^{m+m^{\prime}-1} g_{m, q_{\|}}^{+, f} g_{m^{\prime}, q_{\|}}^{-, f} K_{k k^{\prime} m m^{\prime}}^{f(1)},
\end{aligned}
$$

with 


$$
\begin{aligned}
K_{k k^{\prime} m m^{\prime}}^{\lambda, f(0)}= & \int_{u v w} \exp \left[-w^{2}\right] \exp \left[-u^{2}-u \cdot w+i s_{f}\left(u_{1} w_{2}-u_{2} w_{1}\right)\right] L_{k_{\lambda}}\left(u^{2}\right) L_{m_{\lambda}}\left[(u+w)^{2}\right] \\
& \times \exp \left[-v^{2}-v \cdot w+i s_{f}\left(v_{1} w_{2}-v_{2} w_{1}\right)\right] L_{k_{\lambda}^{\prime}}\left(v^{2}\right) L_{m_{\lambda}^{\prime}}\left[(v+w)^{2}\right] \\
& \times \exp \left\{\frac{i s_{f}}{2}\left[r_{1}\left(2 w_{2}+u_{2}+v_{2}\right)-r_{2}\left(2 w_{1}+u_{1}+v_{1}\right)\right]\right\}, \\
K_{k k^{\prime} m m^{\prime}}^{f(1)}= & -\int_{u v w} \exp \left[-w^{2}\right] \exp \left[-u^{2}-u \cdot w+i s_{f}\left(u_{1} w_{2}-u_{2} w_{1}\right)\right] L_{k-1}^{1}\left(u^{2}\right) L_{m_{+}}\left[(u+w)^{2}\right] \\
& \times(u \cdot v) \exp \left[-v^{2}-v \cdot w+i s_{f}\left(v_{1} w_{2}-v_{2} w_{1}\right)\right] L_{k^{\prime}-1}\left(v^{2}\right) L_{m_{-}^{\prime}}\left[(v+w)^{2}\right] \\
& \times \exp \left\{\frac{i s_{f}}{2}\left[r_{1}\left(2 w_{2}+u_{2}+v_{2}\right)-r_{2}\left(2 w_{1}+u_{1}+v_{1}\right)\right]\right\} .
\end{aligned}
$$

Notice that $K_{k k^{\prime} m m^{\prime}}^{\lambda, f(0)}$ and $K_{k k^{\prime} m m^{\prime}}^{f(1)}$ do not depend on the magnetic field, but they do depend on the external momenta $r_{\perp}=\left(r_{1}, r_{2}\right)$. Thanks to rotational symmetry we can choose $r_{1}=r$ and $r_{2}=0$. Using the polar coordinates

$$
\begin{array}{lll}
u_{1}=u \cos (\alpha-\beta), & v_{1}=v \cos (\alpha-\gamma), & w_{1}=w \cos (\alpha), \\
u_{2}=u \sin (\alpha-\beta), & v_{2}=v \sin (\alpha-\gamma), & w_{2}=w \sin (\alpha),
\end{array}
$$

and performing a series expansion around $r=0$ in the exponential, we can integrate the variable $\alpha$. As seen from Eq. (33), only the terms quadratic in the external momenta $t_{\perp}^{2}$ will contribute to the perpendicular renormalization constant. Thus, we have

$$
\begin{aligned}
& \int_{0}^{2 \pi} d \alpha \exp \left\{i s_{f} r[2 w \sin \alpha+u \sin (\alpha-\beta)+v \sin (\alpha-\gamma)] / 2\right\} \\
& \quad=2 \pi-\frac{\pi r^{2}}{8}\left[u^{2}+v^{2}+4 w^{2}+4 u w \cos \beta+4 v w \cos \gamma+2 u v \cos (\beta-\gamma)\right]+\mathcal{O}\left(r^{4}\right) .
\end{aligned}
$$

The calculation of the remaining integrals can be performed with the aid of the useful relations

$$
\begin{gathered}
\frac{1}{2 \pi} \int_{0}^{2 \pi} d \theta L_{n}\left(x^{2}+y^{2}+2 x y \cos \theta\right) \exp [-x y \exp ( \pm i \theta)]=L_{n}\left(x^{2}\right) L_{n}\left(y^{2}\right), \\
\frac{1}{2 \pi} \int_{0}^{2 \pi} d \theta \cos \theta L_{n}\left(x^{2}+y^{2}+2 x y \cos \theta\right) \exp [-x y \exp ( \pm i \theta)]=-\frac{x y}{2}\left[\frac{L_{n}^{1}\left(x^{2}\right) L_{n}^{1}\left(y^{2}\right)}{n+1}+\frac{L_{n-1}^{1}\left(x^{2}\right) L_{n-1}^{1}\left(y^{2}\right)}{n}\right], \\
\frac{1}{2 \pi} \int_{0}^{2 \pi} d \theta \sin \theta L_{n}\left(x^{2}+y^{2}+2 x y \cos \theta\right) \exp [-x y \exp ( \pm i \theta)]=\mp \frac{i x y}{2}\left[\frac{L_{n}^{1}\left(x^{2}\right) L_{n}^{1}\left(y^{2}\right)}{n+1}-\frac{L_{n-1}^{1}\left(x^{2}\right) L_{n-1}^{1}\left(y^{2}\right)}{n}\right],
\end{gathered}
$$

together with the orthogonality properties of the generalized Laguerre polynomials. This leads to

$$
\begin{aligned}
I_{k k^{\prime}, q_{\|}}^{\lambda, f(0)}= & \frac{B_{f}^{3}}{128 \pi^{3}}\left\{g_{k, q_{\|}}^{\lambda, f} g_{k, q_{\|}}^{\lambda, f} \delta_{k k^{\prime}}-\frac{t_{\perp}^{2}}{4 B_{f}}\left[\left[\left(2 k_{\lambda}+1\right) g_{k, q_{\|}}^{\lambda, f} g_{k, q_{\|}}^{\lambda, f}+\left(k_{\lambda}+1\right) g_{k, q_{\|}}^{\lambda, f} g_{k+1, q_{\|}}^{\lambda, f}\right.\right.\right. \\
& +k_{\lambda} g_{k, q_{\|}}^{\lambda, f} g_{k-1, q_{\|}}^{\lambda, f} \delta_{k k^{\prime}}-\frac{k_{\lambda}+1}{2}\left(g_{k, q_{\|}}^{\lambda, f}+g_{k+1, q_{\|}}^{\lambda, f}\right)^{2} \delta_{k k^{\prime}-1} \\
& \left.\left.-\frac{k_{\lambda}}{2}\left(g_{k, q_{\|}}^{\lambda, f}+g_{k-1, q_{\|}}^{\lambda, f}\right)^{2} \delta_{k k^{\prime}+1}\right]+\mathcal{O}\left(r^{4}\right)\right\}
\end{aligned}
$$

and 


$$
\begin{aligned}
I_{k k^{\prime}, q_{\|}}^{f(1)}= & \frac{k B_{f}^{4}}{32 \pi^{3}}\left\{g_{k, q_{\|}}^{+, f} g_{k, q_{\|}}^{-, f} \delta_{k k^{\prime}}+\frac{t_{\perp}^{2}}{8 B_{f}}\left[\left[-s_{f}\left(g_{k-1, q_{\|}}^{+, f} g_{k+1, q_{\|}}^{+, f}-g_{k-1, q_{\|}}^{-, f} g_{k+1, q_{\|}}^{-, f}\right)\right.\right.\right. \\
& \left.-k\left(4 g_{k, q_{\|}}^{+, f} g_{k, q_{\|}}^{-, f}+g_{k-1, q_{\|}}^{+, f} g_{k, q_{\|}}^{-, f}+g_{k, q_{\|}}^{+, f} g_{k+1, q_{\|}}^{-, f}+g_{k+1, q_{\|}}^{+, f} g_{k, q_{\|}}^{-, f}+g_{k, q_{\|}}^{+, f} g_{k-1, q_{\|}}^{-, f}\right)\right] \delta_{k k^{\prime}} \\
& +(k+1)\left(g_{k, q_{\|}}^{+, f}+g_{k+1, q_{\|}}^{+, f}\right)\left(g_{k, q_{\|}}^{-, f}+g_{k+1, q_{\|}}^{-, f}\right) \delta_{k k^{\prime}-1} \\
& \left.\left.+(k-1)\left(g_{k-1, q_{\|}}^{+, f}+g_{k, q_{\|}}^{+, f}\right)\left(g_{k-1, q_{\|}}^{-, f}+g_{k, q_{\|}}^{-, f}\right) \delta_{k k^{\prime}+1}\right]+\mathcal{O}\left(r^{4}\right)\right\}
\end{aligned}
$$

Replacing the results in Eqs. (A9) and (A10) into Eq. (A1) one arrives at our final expression, quoted in Eq. (41).

\section{APPENDIX B: CALCULATION OF CONTRIBUTIONS TO $f_{\pi^{0}}^{(A \perp)}$}

To calculate the contributions $t_{\perp} \cdot F_{\perp}^{A,(\mathrm{I})}$ and $t_{\perp} \cdot F_{\perp}^{A,(\mathrm{III})}$ to $f_{\pi^{0}}^{(A \perp)}$ we start by integrating $t_{\perp} \cdot h_{\perp}$ along the straight line path $\ell_{\mu}=\beta z_{\mu}$. One has

$$
t_{\perp} \cdot h_{\perp}=-i(2 \pi)^{4} \int_{0}^{1} d \beta \delta^{(2)}\left(r_{\|}-(1-\beta) t_{\|}\right) \partial_{\beta} \delta^{(2)}\left(r_{\perp}-(1-\beta) t_{\perp}\right) .
$$

Given the definition in Eq. (47), we perform a series expansion around $t_{\perp}=0$ up to order $t_{\perp}^{2}$ for each contribution to the axial perpendicular decay constant, similar to the case of $Z_{\perp}$. Thus, we find

$$
\begin{aligned}
& t_{\perp} \cdot F_{\perp}^{A,(\mathrm{I})}(t)=\frac{i N_{C}}{2} t_{\perp}^{2} \sum_{f=u, d} \int_{q} \operatorname{tr}_{D}\left[\tilde{S}_{f}(q)\right] \int_{0}^{1} d \beta \beta\left[g^{\prime}\left(q_{\perp}^{2}+q_{\beta \|}^{+2}\right)+q_{\perp}^{2} g^{\prime \prime}\left(q_{\perp}^{2}+q_{\beta \|}^{+2}\right)\right] \\
& t_{\perp} \cdot F_{\perp}^{A,(\mathrm{II})}(t)=-8 \pi^{2} N_{C} \sum_{f=u, d} \frac{s_{f}}{B_{f}^{3}} \int_{p_{\perp} p_{\perp}^{\prime} q} g\left(q^{2}\right) \exp \left[i 2 \varphi_{0}\left(q_{\perp}, p_{\perp}, p_{\perp}^{\prime}\right) /\left(q_{f} B\right)\right] \\
& \times \operatorname{tr}_{D}\left[\tilde{S}_{f}\left(p_{\perp}, q_{\|}^{+}\right) \gamma_{5}\left(t_{\perp} \cdot \gamma_{\mu}\right) \tilde{S}_{f}\left(p_{\perp}^{\prime}, q_{\|}^{-}\right) \gamma_{5}\right]\left|t_{\perp} \times\left(q_{\perp}-u_{\perp}\right)\right| \\
& t_{\perp} \cdot F_{\perp}^{A,(\mathrm{III})}(t)=-i 4 \pi^{2} N_{C} \sigma \sum_{f=u, d} \frac{1}{B_{f}^{2}} \int_{p_{\perp} p_{\perp}^{\prime} q} g\left(q^{2}\right) \exp \left[i 2 \varphi_{0}\left(q_{\perp}, p_{\perp}, p_{\perp}^{\prime}\right) /\left(q_{f} B\right)\right] \\
& \times \operatorname{tr}_{D}\left[\tilde{S}_{f}\left(p_{\perp}, q_{\|}^{+}\right) \gamma_{5} \tilde{S}_{f}\left(p_{\perp}^{\prime}, q_{\|}^{-}\right) \gamma_{5}\right] \times \sum_{i=1}^{3} f_{i}\left(q_{\perp}, u_{\perp}, t_{\perp}\right)
\end{aligned}
$$

where

$$
\begin{gathered}
f_{1}=-\frac{2 i}{B_{f}}\left|t_{\perp} \times\left(q_{\perp}-u_{\perp}\right)\right|\left(u_{\perp} \cdot t_{\perp}\right) \int_{0}^{1} d \beta\left[g^{\prime}\left(u_{\perp}^{2}+q_{\beta \|}^{-2}\right)-g^{\prime}\left(u_{\perp}^{2}+q_{\beta \|}^{+2}\right)\right], \\
f_{2}=2\left(u_{\perp} \cdot t_{\perp}\right)^{2} \int_{0}^{1} d \beta\left[(1-\beta) g^{\prime \prime}\left(u_{\perp}^{2}+q_{\beta \|}^{-2}\right)+\beta g^{\prime \prime}\left(u_{\perp}^{2}+q_{\beta \|}^{+2}\right)\right], \\
f_{3}=t_{\perp}^{2} \int_{0}^{1} d \beta\left[(1-\beta) g^{\prime}\left(u_{\perp}^{2}+q_{\beta \|}^{-2}\right)+\beta g^{\prime}\left(u_{\perp}^{2}+q_{\beta \|}^{+2}\right)\right] .
\end{gathered}
$$

In these expressions we use the notation $q_{\beta \|}^{+}=q_{\|}+\beta t_{\|} / 2, \quad q_{\beta \|}^{-}=q_{\|}-(1-\beta) t_{\|} / 2, \quad u_{\perp}=p_{\perp}+p_{\perp}^{\prime}-q_{\perp}$, and $\varphi_{0}\left(q_{\perp}, p_{\perp}, p_{\perp}^{\prime}\right)=\varphi\left(q_{\perp}, p_{\perp}, p_{\perp}^{\prime}, 0\right)$.

To calculate the integrals over perpendicular momenta we follow a similar procedure as that described in Appendix A. That is, we introduce the Laguerre-Fourier transforms of the form factors and the expressions for the traces. Afterwards, performing appropriate changes of the integration variables, the integrals can be calculated using the orthogonality properties of the generalized Laguerre polynomials, the properties in Eqs. (A6)-(A8), and the relations 


$$
\begin{aligned}
& \frac{1}{2 \pi} \int_{0}^{2 \pi} d \theta \cos (2 \theta) L_{n}\left(x^{2}+y^{2}+2 x y \cos \theta\right) \exp \left[-x y e^{ \pm i \theta}\right]=\frac{1}{2}\left[F_{n}(x, y)+F_{n-2}(x, y)\right], \\
& \frac{1}{2 \pi} \int_{0}^{2 \pi} d \theta \sin (2 \theta) L_{n}\left(x^{2}+y^{2}+2 x y \cos \theta\right) \exp \left[-x y e^{i \pm \theta}\right]=\frac{ \pm i}{2}\left[F_{n}(x, y)-F_{n-2}(x, y)\right],
\end{aligned}
$$

and

$$
\begin{aligned}
& \int_{0}^{\infty} d w^{2} e^{-w^{2}}\left[F_{m}(x, w) \pm F_{m-2}(x, w)\right]\left[F_{n}(y, w) \pm F_{n-2}(y, w)\right] \\
& =\left(\delta_{m n} \pm \delta_{m n-2}\right) F_{m}(x, y)+\left(\delta_{m n} \pm \delta_{m n+2}\right) F_{m-2}(x, y), \\
& \int_{0}^{\infty} d w^{2} e^{-w^{2}}\left[F_{m}(x, w) \pm F_{m-2}(x, w)\right]\left[F_{n}(y, w) \mp F_{n-2}(y, w)\right] \\
& =\left(\delta_{m n} \mp \delta_{m n-2}\right) F_{m}(x, y)-\left(\delta_{m n} \mp \delta_{m n+2}\right) F_{m-2}(x, y),
\end{aligned}
$$

where

$$
F_{n}(x, y)=L_{n+1}\left(x^{2}\right) L_{n+1}\left(y^{2}\right)-\frac{1}{n+2} L_{n+1}^{1}\left(x^{2}\right) L_{n+1}^{1}\left(y^{2}\right)+\frac{1}{n+1} L_{n}^{1}\left(x^{2}\right) L_{n}^{1}\left(y^{2}\right) .
$$

In the case of calculations of $t_{\perp} \cdot F_{\perp}^{A,(\mathrm{II})}(t)$, and $f_{\pi^{0}}^{(V)}$, some relations between the Bessel functions $J_{\nu}(x)$ and the Laguerre polynomials are also required (see the appendices in Ref. [24]).

In this way, after a lengthy calculation, one arrives at the expression for $f_{\pi^{0}}^{(A \perp)}$ in Eq. (64).

[1] D. E. Kharzeev, K. Landsteiner, A. Schmitt, and H. U. Yee, Lect. Notes Phys. 871, 1 (2013).

[2] J. O. Andersen, W. R. Naylor, and A. Tranberg, Rev. Mod. Phys. 88, 025001 (2016).

[3] V. A. Miransky and I. A. Shovkovy, Phys. Rep. 576, 1 (2015).

[4] D. E. Kharzeev, L. D. McLerran, and H. J. Warringa, Nucl. Phys. A803, 227 (2008); V. Skokov, A. Y. Illarionov, and V. Toneev, Int. J. Mod. Phys. A 24, 5925 (2009); V. Voronyuk, V. Toneev, W. Cassing, E. Bratkovskaya, V. Konchakovski, and S. Voloshin, Phys. Rev. C 83, 054911 (2011).

[5] R. C. Duncan and C. Thompson, Astrophys. J. 392, L9 (1992);

C. Kouveliotou et al., Nature (London) 393, 235 (1998).

[6] M. Chernodub, Phys. Rev. D 82, 085011 (2010).

[7] R. Gatto and M. Ruggieri, Phys. Rev. D 83, 034016 (2011).

[8] K. Fukushima and J. M. Pawlowski, Phys. Rev. D 86, 076013 (2012).

[9] E. Fraga, B. Mintz, and J. Schaffner-Bielich, Phys. Lett. B 731, 154 (2014).

[10] S. Fayazbakhsh and N. Sadooghi, Phys. Rev. D 88, 065030 (2013).

[11] S. Fayazbakhsh, S. Sadeghian, and N. Sadooghi, Phys. Rev. D 86, 085042 (2012).

[12] S. S. Avancini, W. R. Tavares, and M. B. Pinto, Phys. Rev. D 93, 014010 (2016).
[13] R. Zhang, W. j. Fu, and Y.x. Liu, Eur. Phys. J. C 76, 307 (2016).

[14] S. S. Avancini, R. L. S. Farias, M. B. Pinto, W. R. Tavares, and V. S. Timoteo, Phys. Lett. B 767, 247 (2017).

[15] S. S. Avancini, R. L. S. Farias, and W. R. Tavares, Phys. Rev. D 99, 056009 (2019).

[16] S. Mao and Y. Wang, Phys. Rev. D 96, 034004 (2017).

[17] H. Liu, X. Wang, L. Yu, and M. Huang, Phys. Rev. D 97, 076008 (2018).

[18] J. O. Andersen, J. High Energy Phys. 10 (2012) 005.

[19] N. O. Agasian and I. A. Shushpanov, J. High Energy Phys. 10 (2001) 006.

[20] V. D. Orlovsky and Y. A. Simonov, J. High Energy Phys. 09 (2013) 136.

[21] M. A. Andreichikov, B. O. Kerbikov, E. V. Luschevskaya, Y. A. Simonov, and O. E. Solovjeva, J. High Energy Phys. 05 (2017) 007.

[22] B. B. Brandt, G. Bali, G. Endrődi, and B. Glässle, Proc. Sci., LATTICE2015 (2016) 007 [arXiv:1510.03899].

[23] G. S. Bali, B. B. Brandt, G. Endrődi, and B. Glässle, Phys. Rev. D 97, 034505 (2018).

[24] D. Gomez Dumm, M. F. Izzo Villafañe, and N. N. Scoccola, Phys. Rev. D 97, 034025 (2018).

[25] G. Ripka, Quarks Bound by Chiral Fields, (Oxford University, New York, 1997). 
[26] T. Schäfer and E. V. Shuryak, Rev. Mod. Phys. 70, 323 (1998).

[27] C. D. Roberts and A. G. Williams, Prog. Part. Nucl. Phys. 33, 477 (1994); C. D. Roberts and S. M. Schmidt, Prog. Part. Nucl. Phys. 45, S1 (2000).

[28] S. Noguera and N. N. Scoccola, Phys. Rev. D 78, 114002 (2008).

[29] R. D. Bowler and M. C. Birse, Nucl. Phys. A582, 655 (1995); R. S. Plant and M. C. Birse, Nucl. Phys. A628, 607 (1998).

[30] S. M. Schmidt, D. Blaschke, and Y. L. Kalinovsky, Phys. Rev. C 50, 435 (1994).

[31] B. Golli, W. Broniowski, and G. Ripka, Phys. Lett. B 437, 24 (1998); W. Broniowski, B. Golli, and G. Ripka, Nucl. Phys. A703, 667 (2002).

[32] I. General, D. Gomez Dumm, and N. N. Scoccola, Phys. Lett. B 506, 267 (2001); D. Gomez Dumm and N. N. Scoccola, Phys. Rev. D 65, 074021 (2002).

[33] A. Scarpettini, D. Gomez Dumm, and N. N. Scoccola, Phys. Rev. D 69, 114018 (2004).

[34] D. Gomez Dumm, A. G. Grunfeld, and N. N. Scoccola, Phys. Rev. D 74, 054026 (2006).

[35] G. A. Contrera, D. Gomez Dumm, and N. N. Scoccola, Phys. Lett. B 661, 113 (2008).

[36] T. Hell, S. Roessner, M. Cristoforetti, and W. Weise, Phys. Rev. D 79, 014022 (2009); 81, 074034 (2010).

[37] G. A. Contrera, D. Gomez Dumm, and N. N. Scoccola, Phys. Rev. D 81, 054005 (2010).

[38] J. P. Carlomagno, D. Gomez Dumm, and N. N. Scoccola, Phys. Rev. D 88, 074034 (2013); J. P. Carlomagno, Phys. Rev. D 97, 094012 (2018).

[39] D. Gomez Dumm, S. Noguera, and N. N. Scoccola, Phys. Lett. B 698, 236 (2011); Phys. Rev. D 86, 074020 (2012).
[40] V. P. Pagura, D. Gomez Dumm, S. Noguera, and N. N. Scoccola, Phys. Rev. D 95, 034013 (2017).

[41] D. Gomez Dumm, M. F. Izzo Villafañe, S. Noguera, V. P. Pagura, and N. N. Scoccola, Phys. Rev. D 96, 114012 (2017).

[42] G. S. Bali, F. Bruckmann, G. Endrődi, Z. Fodor, S. D. Katz, S. Krieg, A. Schäfer, and K. K. Szabo, J. High Energy Phys. 02 (2012) 044.

[43] G. S. Bali, F. Bruckmann, G. Endrődi, Z. Fodor, S. D. Katz, and A. Schäfer, Phys. Rev. D 86, 071502 (2012).

[44] M. Coppola, D. Gomez Dumm, S. Noguera, and N. N. Scoccola, Phys. Rev. D 99, 054031 (2019).

[45] V. I. Ritus, Sov. Phys. JETP 48, 788 (1978).

[46] P. Watson and H. Reinhardt, Phys. Rev. D 89, 045008 (2014).

[47] N. Mueller, J. A. Bonnet, and C. S. Fischer, Phys. Rev. D 89, 094023 (2014).

[48] M. Coppola, D. Gomez Dumm, S. Noguera, and N. N. Scoccola, Phys. Rev. D 100, 054014 (2019).

[49] K. Fukushima, Phys. Lett. B 591, 277 (2004).

[50] E. Megias, E. Ruiz Arriola, and L. L. Salcedo, Phys. Rev. D 74, 065005 (2006).

[51] C. Ratti, M. A. Thaler, and W. Weise, Phys. Rev. D 73, 014019 (2006).

[52] S. Roessner, C. Ratti, and W. Weise, Phys. Rev. D 75, 034007 (2007).

[53] B. J. Schaefer, M. Wagner, and J. Wambach, Phys. Rev. D 81, 074013 (2010).

[54] B. J. Schaefer, J. M. Pawlowski, and J. Wambach, Phys. Rev. D 76, 074023 (2007).

[55] O. Scavenius, A. Dumitru, and J. T. Lenaghan, Phys. Rev. C 66, 034903 (2002). 